\title{
Coupling impedances of a resistive insert in a vacuum chamber
}

\author{
Yoshihiro Shobuda \\ JAEA, Japan Atomic Energy Agency, 2-4 Shirakata Shirane, Tokaimura, Nakagun, Ibaraki 319-1195, Japan
}

Yong Ho Chin and Koji Takata

KEK, High Energy Accelerator Research Organization, 1-1 Oho, Tsukuba, Ibaraki 305-0801, Japan

(Received 23 April 2009; published 17 September 2009)

\begin{abstract}
We have developed a theory to calculate both longitudinal and transverse impedances of a resistive short (typically shorter than the chamber radius) insert with cylindrical symmetry, sandwiched by perfectly conductive chambers on both sides. It is found that unless the insert becomes extremely thin (typically a few $\mathrm{nm}$ for a metallic insert) the entire image current runs on the thin insert, even in the frequency range where the skin depth exceeds the insert thickness, and therefore the impedance increases drastically from the conventional resistive-wall impedance. In other words, the wakefields do not leak out of the insert unless it is extremely thin. Formulas of the impedance valid for various cases of the insert are categorized in summary.
\end{abstract}

DOI: 10.1103/PhysRevSTAB.12.094401

PACS numbers: 41.75.-i, 29.27.-a

\section{INTRODUCTION}

The impedance of a finite-length, resistive insert in a beam pipe has recently been studied in several reports [14]. To simplify this complicated problem, it is often assumed that the skin depth is smaller than the radius of the chamber and its thickness, and therefore investigations have been limited to the behavior of the impedance in a high frequency region $[2,3]$. In this case, the wakefields inside the chamber are completely shielded and do not leak out of the insert. However, in the frequency region where the skin depth exceeds the chamber thickness, these theories are no longer valid.

In proton synchrotrons, the inner surface of a short ceramic break is normally coated by a thin (typically about $10 \mathrm{~nm}$ ) titanium nitride (TiN) to suppress the secondary emission of electrons. The skin depth can be larger than the thickness of the TiN coating in low frequency, and the wakefields may interact with the outside world through the coating. It is thus important to construct a theory of resistive insert taking into account its thickness effects.

The resistive-wall impedance has been studied for many years and many formulas are obtained [5-8]. But, those formulas are sometimes applicable only to a relativistic beam with a limited frequency range. Recently, Burov and Lebedev, and Metral et al. have calculated the resistivewall impedance of chambers composed of more than one layer [9-11]. Their theories, however, assume a beam pipe with translational symmetry. For a finite-length insert in a beam pipe, a new theory is needed.

Recently, we have developed the theory to describe the impedance of a gap that is sandwiched by perfectly conductive chambers [12]. This theory gives us basic understanding of the interaction between a beam and a gap. Replacing this gap by an insert, we can construct a theory of the impedance of the insert as a three-dimensional problem. The main difference between a gap and an insert is that the insert has a finite skin depth, and this skin depth effect will modulate how wakefields propagate in the chamber and the impedance of the insert.

We consider only cylindrical symmetric problems in this paper. The main objective is to study how the impedance will change from that of the conventional resistive-wall theory to that of a gap when the thickness of the insert is changed compared to the skin depth. We develop a theory to describe the impedance of a short insert with cylindrical symmetry by generalizing the theory of impedance of a gap. In Secs. II and III, we develop the theory of both the longitudinal and transverse impedances, respectively. In these sections, we compare the impedance of the resistive wall with finite thickness and that of the short insert. The paper is summarized in Sec. IV.

In numerical examples shown in the figures, unless specified otherwise, we consider a beam pipe radius $a=$ $5 \mathrm{~cm}$ with an insert of length $g=8 \mathrm{~mm}$, and conductivity $\sigma_{c}=6 \times 10^{6} / \Omega \mathrm{m}$. This can be a model for a short ceramic break with TiN coating in a copper beam pipe.

\section{LONGITUDINAL IMPEDANCE OF A RESISTIVE INSERT IN A BEAM PIPE}

Let us start with deriving electromagnetic fields generated by the interaction between a beam and an insert in the cylindrically symmetric system. We use the cylindrical coordinates $(\rho, \theta, z)$ as shown in Fig. 1. We assume that both the sandwiching chambers and the insert have thickness $t$ and the inner radius of $a$. The insert is located in the region where $-w<z<w$ (namely, the length of the insert $g$ is equal to $2 w$ ).

In order to obtain formal solutions of the fields, we apply the field matching technique to this system. We assume that the beam has the cylindrically uniform density with the 


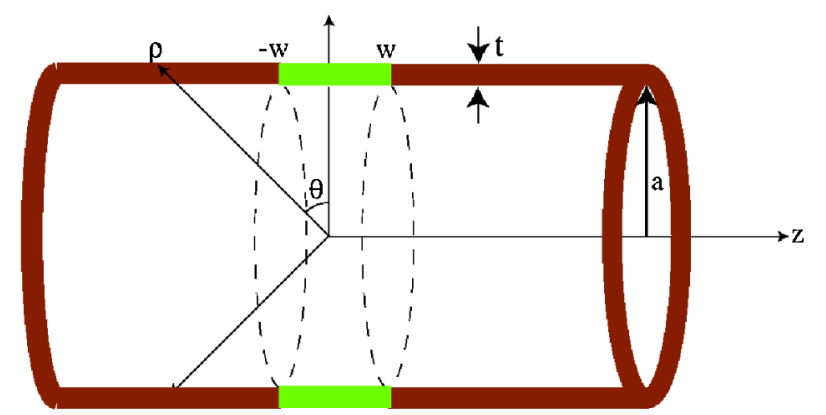

FIG. 1. (Color) The insert (denoted by green objects), which is sandwiched by the perfectly conductive metal chambers and the cylindrical coordinate system $(\rho, \theta, z)$.

radius of $\sigma$ and its total charge is 1C. Namely, its current density is given by

$$
j_{z}=\beta c[1-\Theta(\rho-\sigma)] e^{-j k z+j \omega t} /\left(\pi \sigma^{2}\right),
$$

where $\Theta(x)$ is the step function, $k=\omega / \beta c, \beta=v / c, v$ is the velocity of the beam, $c$ is the velocity of light, $\omega=$ $2 \pi f$, and $f$ is the frequency. The formal solutions of the fields at the frequency $\omega$ inside the chamber are given by (see Appendix A)

$$
\begin{aligned}
E_{z}= & \frac{j k}{\gamma^{2}} \frac{c Z_{0}}{\pi \sigma^{2}}\left(\frac{1}{\bar{k}^{2}}-\frac{\sigma I_{0}(\bar{k} \rho) K_{1}(\bar{k} \sigma)}{\bar{k}}\right) e^{-j k z} \\
& +\int_{-\infty}^{\infty} d h A(h) e^{-j h z} \frac{J_{0}(\Lambda \rho)}{J_{0}(\Lambda a)}, \\
H_{\theta}= & \frac{\beta c}{\pi \sigma} K_{1}(\bar{k} \sigma) I_{1}(\bar{k} \rho) e^{-j k z} \\
& +\frac{j k \beta}{Z_{0}} \int_{-\infty}^{\infty} d h A(h) e^{-j h z} \frac{J_{1}(\Lambda \rho)}{\Lambda J_{0}(\Lambda a)},
\end{aligned}
$$

for $\rho<\sigma$, and

$$
\begin{gathered}
E_{z}=\frac{j c Z_{0}}{\pi \sigma \gamma} I_{1}(\bar{k} \sigma) K_{0}(\bar{k} \rho) e^{-j k z} \\
+\int_{-\infty}^{\infty} d h A(h) e^{-j h z} \frac{J_{0}(\Lambda \rho)}{J_{0}(\Lambda a)}, \\
H_{\theta}=\frac{\beta c}{\pi \sigma} I_{1}(\bar{k} \sigma) K_{1}(\bar{k} \rho) e^{-j k z} \\
+\frac{j k \beta}{Z_{0}} \int_{-\infty}^{\infty} d h A(h) e^{-j h z} \frac{J_{1}(\Lambda \rho)}{\Lambda J_{0}(\Lambda a)},
\end{gathered}
$$

for $\rho>\sigma$, where $\bar{k}=k / \gamma, \gamma$ is the Lorentz factor, $A(h)$ is the expansion coefficient, $Z_{0}(=120 \pi)$ is the impedance of free space, $J_{m}(z)$ is the Bessel function, and $\Lambda=$ $\sqrt{k^{2} \beta^{2}-h^{2}}$. The time dependence of the fields is assumed to be harmonic and it is expressed as the complex exponential $e^{j \omega t}$. Since $E_{z}$ on the inner surface of the chamber should be zero except in the insert, the expansion coefficient $A(h)$ should satisfy the following relation:

$$
\begin{aligned}
& \frac{j c Z_{0}}{\pi \sigma \gamma} I_{1}(\bar{k} \sigma) K_{0}(\bar{k} a) e^{-j k z}+\int_{-\infty}^{\infty} d h A(h) e^{-j h z} \\
& \quad= \begin{cases}\frac{\tilde{V}_{1}}{2 w} & \text { for }-w<z<w \\
0 & \text { otherwise, }\end{cases}
\end{aligned}
$$

where $\tilde{V}_{1}$ is the voltage inside the insert at $\rho=a$. Here the assumption was made that the longitudinal length of the insert $g$ is short (typically shorter than the radius of the chamber) and thus, the $z$ dependence of $E_{z}$ on the insert can be negligible. Then, the expansion coefficient $A(h)$ is rewritten by the insert voltage $\tilde{V}_{1}$ as

$$
\frac{j c Z_{0}}{\pi \sigma \gamma} I_{1}(\bar{k} \sigma) K_{0}(\bar{k} a) \delta(h-k)+A(h)=\frac{\tilde{V}_{1}}{2 \pi} \frac{\sin h w}{h w} .
$$

Substituting Eq. (7) into Eqs. (2)-(5), we obtain

$$
\begin{aligned}
E_{z}= & \frac{j c Z_{0}}{\pi \sigma^{2} \gamma}\left(\frac{1}{\bar{k}}-\sigma I_{0}(\bar{k} \rho) K_{1}(\bar{k} \sigma)\right. \\
& \left.-\frac{\sigma I_{0}(\bar{k} \rho) I_{1}(\bar{k} \sigma) K_{0}(\bar{k} a)}{I_{0}(\bar{k} a)}\right) e^{-j k z} \\
& +\frac{\tilde{V}_{1}}{2 \pi} \int_{-\infty}^{\infty} d h e^{-j h z} \frac{J_{0}(\Lambda \rho)}{J_{0}(\Lambda a)} \frac{\sin h w}{h w},
\end{aligned}
$$

$$
\begin{aligned}
H_{\theta}= & \frac{\beta c}{\pi \sigma}\left(K_{1}(\bar{k} \sigma)+\frac{I_{1}(\bar{k} \sigma) K_{0}(\bar{k} a)}{I_{0}(\bar{k} a)}\right) I_{1}(\bar{k} \rho) e^{-j k z} \\
& +\frac{\tilde{V}_{1}}{2 \pi} \frac{j k \beta}{Z_{0}} \int_{-\infty}^{\infty} d h e^{-j h z} \frac{J_{1}(\Lambda \rho)}{\Lambda J_{0}(\Lambda a)} \frac{\sin h w}{h w},
\end{aligned}
$$

for $\rho<\sigma$, and

$$
\begin{aligned}
E_{z}= & \frac{j c Z_{0}}{\pi \sigma \gamma} I_{1}(\bar{k} \sigma)\left(K_{0}(\bar{k} \rho)-\frac{I_{0}(\bar{k} \rho) K_{0}(\bar{k} a)}{I_{0}(\bar{k} a)}\right) e^{-j k z} \\
& +\frac{\tilde{V}_{1}}{2 \pi} \int_{-\infty}^{\infty} d h e^{-j h z} \frac{J_{0}(\Lambda \rho)}{J_{0}(\Lambda a)} \frac{\sin h w}{h w} \\
H_{\theta}= & \frac{\beta c}{\pi \sigma} I_{1}(\bar{k} \sigma)\left(K_{1}(\bar{k} \rho)+\frac{K_{0}(\bar{k} a) I_{1}(\bar{k} \rho)}{I_{0}(\bar{k} a)}\right) e^{-j k z} \\
& +\frac{\tilde{V}_{1}}{2 \pi} \frac{j k \beta}{Z_{0}} \int_{-\infty}^{\infty} d h e^{-j h z} \frac{J_{1}(\Lambda \rho)}{\Lambda J_{0}(\Lambda a)} \frac{\sin h w}{h w},
\end{aligned}
$$

for $\rho>\sigma$.

For the fields outside the chamber, we follow Silver and Saunders's theory [13] and describe those as (see Appendix B)

$$
\begin{aligned}
E_{z} & =\frac{\tilde{V}_{2}}{2 \pi} \int_{-\infty}^{\infty} \frac{H_{0}^{(2)}(\Lambda \rho)}{H_{0}^{(2)}[\Lambda(a+t)]} \frac{\sin h w}{h w} e^{-j h z} d h, \\
H_{\theta} & =j \frac{\beta k}{Z_{0}} \frac{\tilde{V}_{2}}{2 \pi} \int_{-\infty}^{\infty} \frac{H_{1}^{(2)}(\Lambda \rho)}{\Lambda H_{0}^{(2)}[\Lambda(a+t)]} \frac{\sin h w}{h w} e^{-j h z} d h,
\end{aligned}
$$




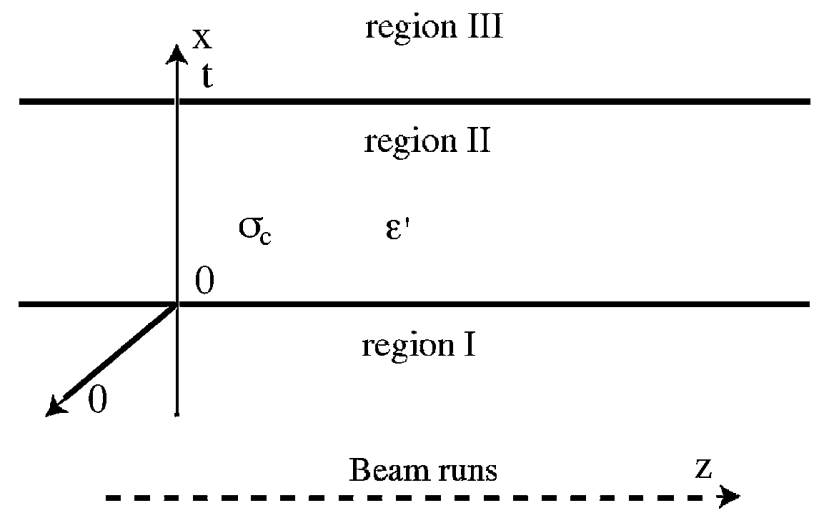

FIG. 2. A wall with thickness $t$, the conductivity of $\sigma_{c}$, and the relative dielectric constant of $\epsilon^{\prime}$. The beam runs in region I.

for $\rho>a+t$, where $H_{m}^{(2)}(z)$ is the Hankel functions of the second kind and $\tilde{V}_{2}$ is the voltage inside the insert at $\rho=$ $a+t$. Here we should notice that Eq. (12) satisfies the condition that it should be zero on the outer surface of the chamber except in the insert. These insert voltages $\tilde{V}_{1}$ and $\tilde{V}_{2}$ will be determined by boundary conditions.

In order to match the solution for $\rho<a$ and that for $\rho>$ $a+t$, we have to find the relation of fields at $\rho=a$ and $\rho=a+t$, especially inside the insert. Hence, as shown in

Fig. 2, we consider the one-dimensional problem of a wall with thickness $t$ in free space. We call region $(x<0)$ region I, $(0<x<t)$ region II, and $(t<x<\infty)$ region III. The beam running in region I creates fields on the inner surface of the wall $(x=0)$, which are written as $E_{z}(0)$ and $H_{\theta}(0)$. If we assume that the insert obeys Ohm's law, $\vec{j}=\sigma_{c} \vec{E}$, Maxwell equations in region II are written as follows:

$$
\begin{gathered}
-\frac{\partial E_{z}}{\partial x}(x)=-j k \beta Z_{0} H_{\theta}(x), \\
\frac{\partial H_{\theta}(x)}{\partial x}=\left(\sigma_{c}+j \frac{k \beta \epsilon^{\prime}}{Z_{0}}\right) E_{z}(x),
\end{gathered}
$$

where $\epsilon^{\prime}$ is the relative dielectric constant of the insert, usually negligible compared to the first term in Eq. (15) [14]. The solutions are

$$
\begin{aligned}
E_{z}(x)= & E_{z}(0) \cosh \sqrt{j k \beta Z_{0}\left(\sigma_{c}+j \frac{k \beta \epsilon^{\prime}}{Z_{0}}\right) x} \\
& +H_{\theta}(0) \frac{j Z_{0} k \beta \sinh \sqrt{j k \beta Z_{0}\left(\sigma_{c}+j \frac{k \beta \epsilon^{\prime}}{Z_{0}}\right)} x}{\sqrt{j k \beta Z_{0}\left(\sigma_{c}+j \frac{k \beta \epsilon^{\prime}}{Z_{0}}\right)}},
\end{aligned}
$$

Since the fields inside the chamber [given by Eqs. (4) and (5)] and outside the chamber [given by Eqs. (12) and (13)] must be connected through the relations (16) and (17), one of the insert voltages $\tilde{V}_{1}$ can be solved as follows:

$$
\tilde{V}_{1}=-\frac{\frac{2 \gamma c Z_{0} I_{1}(\bar{k} \sigma) e^{-j k z}}{j k^{2} \sigma a I_{0}(\bar{k} a)}\left(1+\frac{k^{2} \beta^{2} w \tanh \sqrt{j k \beta Z_{0}\left(\sigma_{c}+j \frac{k \beta \epsilon^{\prime}}{Z_{0}}\right) t}}{\pi \sqrt{j k \beta Z_{0}\left(\sigma_{c}+j \frac{k \beta \epsilon^{\prime}}{Z_{0}}\right)}}\right)}{J+Y-\left(\frac{\pi \sqrt{j k \beta Z_{0}\left(\sigma_{c}+j \frac{k \beta \epsilon^{\prime}}{Z_{0}}\right)}}{k^{2} \beta^{2} w}+\frac{k^{2} \beta^{2} w J Y}{\pi \sqrt{j k \beta Z_{0}\left(\sigma_{c}+j \frac{k \beta \epsilon^{\prime}}{Z_{0}}\right)}}\right) \tanh \sqrt{j k \beta Z_{0}\left(\sigma_{c}+j \frac{k \beta \epsilon^{\prime}}{Z_{0}}\right)} t},
$$

where $J$ and $Y$ are defined as

$$
J=\frac{1}{2 w} \int_{-\infty}^{\infty} d h \int_{-w}^{w} d \xi \frac{e^{-j h(z-\xi)}}{\Lambda} \frac{J_{1}(\Lambda a)}{J_{0}(\Lambda a)}, \quad Y=-\frac{1}{2 w} \int_{-\infty}^{\infty} d h \int_{-w}^{w} d \xi \frac{e^{-j h(z-\xi)}}{\Lambda} \frac{H_{1}^{(2)}[\Lambda(a+t)]}{H_{0}^{(2)}[\Lambda(a+t)]},
$$

$z$ is the matching point that should be inside of the insert, i.e., $-w<z<w$. The average value of $E_{z}$ [expressed by Eq. (2) over $\rho$ ] gives the coupling impedance (see Appendix A 1). Since the condition

$$
\frac{k^{2} \beta^{2} w^{2}}{\sqrt{j k \beta Z_{0}\left(\sigma_{c}+j \frac{k \beta \epsilon^{\prime}}{Z_{0}}\right)} w} \tanh \sqrt{j k \beta Z_{0}\left(\sigma_{c}+j \frac{k \beta \epsilon^{\prime}}{Z_{0}}\right)} t \ll 1
$$

is satisfied in most of the cases, it can be approximately expressed as

$$
Z_{L}=-\frac{j Z_{0}}{k \beta \pi \sigma^{2}}\left(1-\frac{2 K_{0}(\bar{k} a) I_{1}^{2}(\bar{k} \sigma)}{I_{0}(\bar{k} a)}-2 I_{1}(\bar{k} \sigma) K_{1}(\bar{k} \sigma)\right) \mathcal{L}+Z_{\text {insert }, L}
$$




$$
Z_{\text {insert } L} \simeq \frac{4 Z_{0} I_{1}^{2}(\bar{k} \sigma) e^{-j k z}}{j \beta \gamma \sigma^{2} a \bar{k}^{3} I_{0}^{2}(\bar{k} a)\left[J+Y-\frac{\pi \sqrt{j k \beta Z_{0}\left(\sigma_{c}+j \frac{k \epsilon_{\epsilon_{0}^{\prime}}}{Z_{0}}\right)}}{k^{2} \beta^{2} w} \tanh \sqrt{j k \beta Z_{0}\left(\sigma_{c}+j \frac{k \beta \epsilon^{\prime}}{Z_{0}}\right)} t\right]},
$$

where $\mathcal{L}$ is the length of the beam pipe. The first term in Eq. (21) represents the nonrelativistic space charge impedance (see Appendix A 1) [12]. The second term $Z_{\text {insert } L}$ in Eq. (21) is the coupling impedance of the insert.

The integration of the Bessel functions can be done simply by picking up residues in the complex plane $h$. However, the integration of the Hankel functions is a quite complicated and difficult task since there are branch points at $h= \pm k \beta$ in the complex plane $h$. To proceed further, we follow the manipulation explained in Ref. [12]. We first rewrite the integration of the Hankel functions as shown in Eq. (C3). This manipulation enables the integration of the Hankel functions over $h$ with the usual residue theorem. We choose the path of integration to be below the poles for $h<0$ and above the poles for $h>0$. Finally, we obtain the expression of the longitudinal coupling impedance as

$$
Z_{L, \text { insert }}=\frac{4 Z_{0} I_{1}^{2}(\bar{k} \sigma) e^{-j k z}}{j \beta \gamma \sigma^{2} a \bar{k}^{3} I_{0}^{2}(\bar{k} a)\left[Y_{\mathrm{pole}}+Y_{\mathrm{cut}}-\frac{\pi \sqrt{j k \beta Z_{0}\left(\sigma_{c}+j \frac{k \beta \epsilon^{\prime}}{Z_{0}}\right)}}{k^{2} \beta^{2} w} \tanh \sqrt{j k \beta Z_{0}\left(\sigma_{c}+j \frac{k \beta \epsilon^{\prime}}{Z_{0}}\right)} t\right]},
$$

where

$$
\begin{aligned}
& Y_{\text {pole }}=-\sum_{s=1}^{\infty} \frac{\pi a\left(2-e^{-j\left(b_{s} / a\right)(z+w)}-e^{j\left(b_{s} / a\right)(z-w)}\right)}{w b_{s}^{2}}, \\
& Y_{\text {cut }}=-\frac{1}{w \pi(a+t)} \int_{0}^{\infty} d \zeta \frac{2-e^{-j(z+w) \sqrt{k^{2} \beta^{2}+\frac{\zeta}{(a+t)^{2}}}}-e^{j(z-w) \sqrt{k^{2} \beta^{2}+\frac{\zeta}{(a+t)^{2}}}}}{\zeta\left(k^{2} \beta^{2}+\frac{\zeta}{(a+t)^{2}}\right) H_{0}^{(1)}\left(e^{j(\pi / 2)} \sqrt{\zeta}\right) H_{0}^{(2)}\left(e^{j(\pi / 2)} \sqrt{\zeta}\right)} \simeq \frac{2(1-j)}{\sqrt{k \beta w}} .
\end{aligned}
$$

Here, $b_{s}^{2}=k^{2} \beta^{2} a^{2}-j_{0, s}^{2}=-\beta_{s}^{2}, j_{0, s}$ are $s$ th zeros of $J_{0}(z)$ and $H_{m}^{(1)}(z)$ is the Hankel function of the first kind. We should notice that $b_{s}$ approaches $-j \beta_{s}$ for $j_{0, s}>k \beta a$. In the above derivation, we used Eqs. (C1) and (C3) of Appendix C. The integration in Eq. (25) over $\zeta$ is much more straightforward than the integration in Eq. (22) over $h$, since there is no singular point except $\zeta=0$ along the integration path.

\section{A. Frequency dependence and length dependence of the impedance}

In this subsection, we assume that the chamber thickness $t$ satisfies the condition

$$
t>t_{\min } \equiv\left(\frac{4 g}{\pi^{2} Z_{0}^{3} \sigma_{c}^{3}}\right)^{1 / 4}
$$

We exclude an extremely thin insert case here. This assumption allows us to neglect the effect from radiation terms such as $Y_{\text {pole }}$ and $Y_{\text {cut }}$ in Eq. (23) in the low frequency region where the skin depth exceeds the insert thickness $t$. The thickness $t_{\min }$ is typically a few $10 \mathrm{~nm}$ for a metallic insert.

Krinsky et al. and Stupakov [2,3] studied the impedance of a short insert. Their results indicate that when $g \ll$ $\left(Z_{0} \sigma a^{4} / 4 \pi\right)^{1 / 3}$ and

$$
f \gg f_{D} \equiv \frac{c}{2 \pi} \sqrt{\frac{2 Z_{0} \sigma_{c}}{g}},
$$

(the frequency $f_{D}$ is typically of the order of $\mathrm{THz}$ in our short insert), then

$$
Z_{L} \simeq \frac{(1-j) 2 Z_{0 \sqrt{g}}}{2 \pi a \sqrt{\pi k}},
$$

and is proportional to $\sqrt{g}$.

Let us consider the case that the thickness of the insert is larger than $2^{1 / 2} \pi^{3 / 4} t_{\min }$ and see if our theory can reproduce Eq. (28) in the extremely high frequency region $f \gg f_{D}$. In this frequency region, we may take a limit of $t$ to infinity in Eq. (23), and the following inequality can be applied to Eq. (23):

$$
\left|Y_{\text {cut }}\right| \gg\left|\frac{\pi \sqrt{j k \beta Z_{0} \sigma_{c}}}{k^{2} \beta^{2} w}+Y_{\text {pole }}\right| .
$$

Then, Eq. (23) becomes

$$
Z_{L, \text { insert }} \simeq \frac{(1-j) 2 Z_{0 \sqrt{g}}}{2 \pi a I_{0}^{2}(\bar{k} a) \sqrt{\beta \pi k}},
$$

by choosing the matching point $z$ as zero for an extremely thin beam (the beam radius $\sigma=0$ ). Specifically for a relativistic beam, Eq. (30) reproduces Eq. (28). These results show that the impedance decreases in proportion 
to $k^{-1 / 2}$ in the extremely high frequency, as predicted by the diffraction theory [15].

In the intermediate region of $f \ll f_{D}$ where the skin depth $\delta$ is still smaller than the insert thickness $t$, we can apply the following inequality to Eq. (23):

$$
\left|Y_{\text {pole }}+Y_{\text {cut }}\right| \ll\left|\frac{\pi \sqrt{j k \beta Z_{0} \sigma_{c}}}{k^{2} \beta^{2} w}\right| .
$$

We then obtain the conventional formula of the resistivewall impedance for a relativistic beam [7]:

$$
Z_{L, \text { insert }} \simeq g Z_{0} \sqrt{\frac{2 \omega}{c Z_{0} \sigma_{c}}} \frac{1+j}{4 \pi a},
$$

which is proportional to the length of the insert $g$.

In the low frequency region where the skin depth exceeds the insert thickness $t$,

$$
f<f_{\delta} \equiv \frac{c}{\pi Z_{0} \sigma_{c} t^{2}},
$$

but the effect from radiation terms such as $Y_{\text {pole }}$ and $Y_{\text {cut }}$ are still negligible in Eq. (23), we obtain

$$
Z_{L, \text { insert }} \simeq \frac{g}{2 \pi a \sigma_{c} t} .
$$

When the thickness of the insert is smaller than $2^{1 / 2} \pi^{3 / 4} t_{\min }$ but larger than $t_{\min }$, Eq. (34) becomes valid all the way up to $f_{D}$.

\section{B. Dependence of the insert impedance on its thickness}

Before studying the thickness dependence of the insert impedance, let us study the thickness dependence of the resistive-wall impedance in order to compare them with our results afterwards. We numerically calculate the resistive-wall impedance for different thicknesses of the chamber by borrowing the general formulas of the resistive-wall impedance with finite thickness from Metral et al.'s recent work [10] (see Appendix A 2):

$$
Z_{L}=-\frac{j Z_{0} g \bar{k}}{2 \pi \beta \gamma}\left(\frac{K_{0}(\bar{k} a)}{I_{0}(\bar{k} a)}+C_{3}\right)
$$

where

$$
\begin{gathered}
C_{3}=\frac{\left[\sigma_{c} Z_{0} K_{0}(\bar{k} a) I_{1}\left(\nu_{2} a\right)+j \beta \gamma \nu_{2} K_{1}(\bar{k} a) I_{0}\left(\nu_{2} a\right)\right] \alpha-\sigma_{c} Z_{0} K_{0}(\bar{k} a) K_{1}\left(\nu_{2} a\right)+j \beta \gamma \nu_{2} K_{1}(\bar{k} a) K_{0}\left(\nu_{2} a\right)}{\left[-\sigma_{c} Z_{0} I_{0}(\bar{k} a) I_{1}\left(\nu_{2} a\right)+j \beta \gamma \nu_{2} I_{1}(\bar{k} a) I_{0}\left(\nu_{2} a\right)\right] \alpha+\sigma_{c} Z_{0} I_{0}(\bar{k} a) K_{1}\left(\nu_{2} a\right)+j \beta \gamma \nu_{2} I_{1}(\bar{k} a) K_{0}\left(\nu_{2} a\right)}, \\
\alpha=\frac{\sigma_{c} Z_{0} K_{1}\left[\nu_{2}(a+t)\right] K_{0}[\bar{k}(a+t)]-j \beta \gamma \nu_{2} K_{0}\left[\nu_{2}(a+t)\right] K_{1}[\bar{k}(a+t)]}{\sigma_{c} Z_{0} I_{1}\left[\nu_{2}(a+t)\right] K_{0}[\bar{k}(a+t)]+j \beta \gamma \nu_{2} I_{0}\left[\nu_{2}(a+t)\right] K_{1}[\bar{k}(a+t)]},
\end{gathered}
$$

where $\nu_{2}=\sqrt{k^{2}+j k \beta Z_{0} \sigma_{c}}$. The results for a relativistic beam are shown in Fig. 3. The red, the blue, and the black lines show the cases that the insert thickness $t$ is equal to infinity, $10 \mu \mathrm{m}$ and $1 \mu \mathrm{m}$, respectively. The impedance starts to deviate from that for the infinitely thick chamber when the skin depth exceeds the chamber thickness. Apparently, the wakefields leak out at low frequency. The dependence of $Z_{L}$ on the conductivity $\sigma_{c}$, the frequency $f$, and the chamber thickness $t$, for the case that the skin depth exceeds $t$, can be approximately written as

$$
\Re\left[Z_{L}\right] \simeq \frac{2 g \pi Z_{0}^{2} \sigma_{c} f^{2} t^{3}}{3 a c^{2}} .
$$

Contrary to our intuition, the impedance becomes larger as the conductivity of the material $\sigma_{c}$ increases.

Now, let us discuss the properties of the impedance of the insert by changing the thickness of it. The thickness dependence of the real part of the insert impedance obtained by Eq. (23) is shown in Fig. 4. The red, the blue, the black, the black dashed, the black dot, the green and the blue dashed, and the red dashed lines represent the cases that the thickness $t$ is equal to $100 \mu \mathrm{m}, 10 \mu \mathrm{m}, 1 \mu \mathrm{m}$, $100 \mathrm{~nm}, 10 \mathrm{~nm}, 1 \mathrm{~nm}, 100 \mathrm{pm}$, and $10 \mathrm{pm}$, respectively. When the skin depth is smaller than the thickness of the insert but the frequency $f$ is lower than $f_{D}$, the impedance of the insert is identical to the resistive-wall impedance given by Eq. (32) (see the results for the case that $t$ is equal to $100 \mu \mathrm{m}$ ). As we find from the result of $t=10 \mu \mathrm{m}$ in Fig. 4 , if the skin depth exceeds the insert thickness $\left[f_{\delta} \simeq\right.$ $0.42 \mathrm{GHz}$ in this case; see Eq. (33)], the real part of the impedance becomes independent of the frequency. The imaginary part is still inductive for the insert with this thickness. This indicates that the whole wall current runs in the thin insert, despite the fact that the skin depth exceeds the insert thickness in most of the frequencies. In other words, the beam current is completely shielded by the wall current in the insert, and the wakefields do not propagate out of the chamber. If this picture is correct, the real part of impedance should be equal to the resistance of the wall current $Z_{\text {wall }}$. Actually, the results of $t=1 \mu \mathrm{m}$ to $t=100 \mathrm{~nm}$ (even including the result of $10 \mathrm{~nm}$ that is smaller than $t_{\text {min }}$ ) described in Fig. 4 are equal to the resistance of the wall current $Z_{\text {wall }}$ :

$$
Z_{\text {wall }}=\frac{g}{\sigma_{c} \pi\left[(a+t)^{2}-a^{2}\right]} \simeq \frac{g}{2 \pi a \sigma_{c} t} .
$$

This behavior of the insert impedance is quite different from that of the resistive-wall impedance of the chamber with finite thickness for a relativistic beam, which was discussed in the first paragraph of this subsection.

When the thickness of the insert is extremely thin like $t \ll t_{\min }$, the situation is quite different from the above 


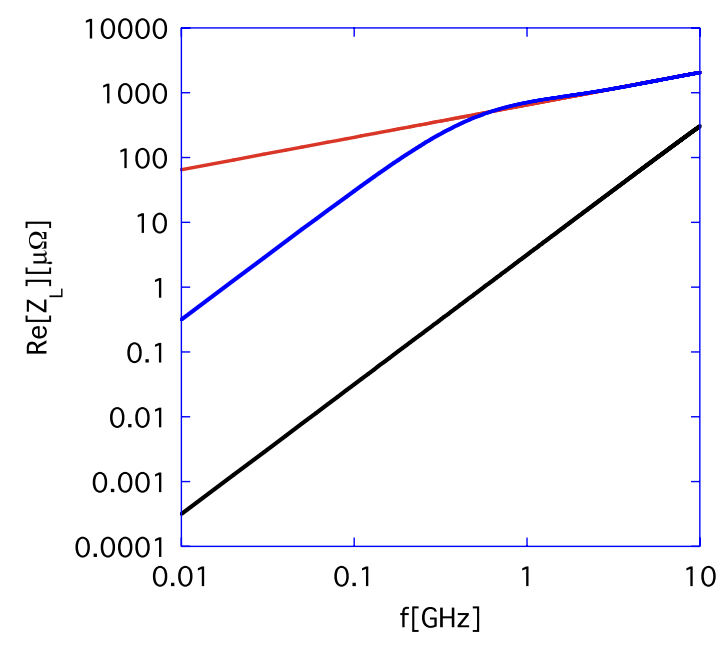

FIG. 3. (Color) The dependence of the real part of the longitudinal resistive-wall impedance of a uniform beam pipe (no insert) on the thickness of the chamber. The length of the beam pipe $g=8 \mathrm{~mm}$. Lorentz factor $\gamma=1000$. The red, the blue, and the black lines show the cases that the chamber thickness $t$ is equal to infinity, 10 and $1 \mu \mathrm{m}$, respectively. The impedance for finite thickness starts to deviate from that for infinite thickness at the frequency where the skin depth exceeds the chamber thickness.

case. The results of $t=1 \mathrm{~nm}$ to $t=10 \mathrm{pm}$ in Fig. 4 correspond to this case. The frequency $f_{D}$ and the skin depth $\delta$ are no longer dominant parameters. The new parameter,

$$
f_{c} \equiv \frac{\sigma_{c}^{2} Z_{0}^{2} t^{2} c}{4 \pi g},
$$

plays a more important role in the impedance. In the

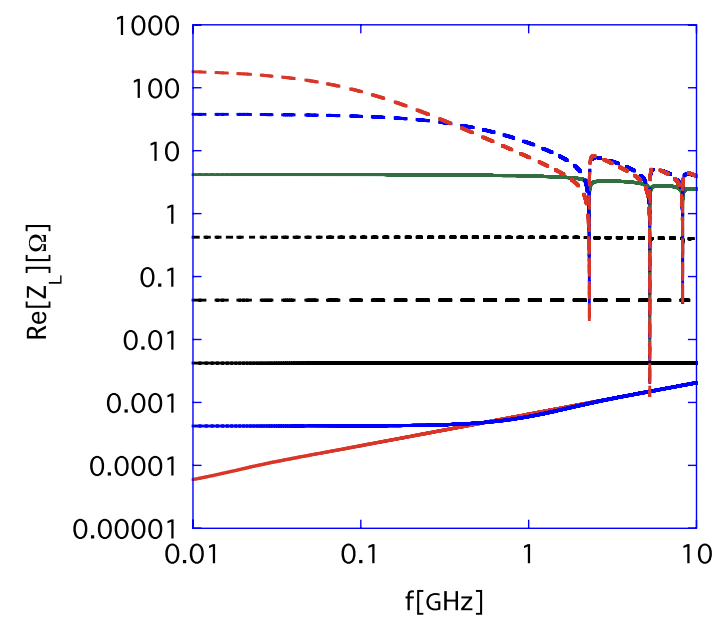

FIG. 4. (Color) The thickness dependence of the longitudinal impedance of the insert in the relativistic beam case. The red, the blue, the black, the black dashed, the black dot, the green, and the blue dashed and the red dashed lines represent the cases that thickness $t$ is equal to $100 \mu \mathrm{m}, 10 \mu \mathrm{m}, 1 \mu \mathrm{m}, 100 \mathrm{~nm}, 10 \mathrm{~nm}$, $1 \mathrm{~nm}, 100 \mathrm{pm}$, and $10 \mathrm{pm}$, respectively. The matching point is $z=0$. frequency region $f \ll f_{c}$, the contribution from the wall current dominates in the impedance. In the rest of the frequency, the radiation effects become dominant contributions. The dips for these cases in Fig. 4 correspond to the cutoff frequencies of the chamber. The imaginary part of impedance becomes capacitive, which is opposite to the result of $t>t_{\min }$.

Figure 5 represents the dependence of the impedance on the length of the insert $g$ when the thickness of the insert $t$ is equal to $1 \mathrm{~nm}$ and Lorentz factor $\gamma$ is equal to 1000 . The red, the blue, and the black lines show the longitudinal impedances per a unit length for the cases that the length of the insert $g$ is equal to $2 \mathrm{~mm}, 8 \mathrm{~mm}$, and $18 \mathrm{~mm}$, respectively. The impedances themselves (not normalized by the gap length $g$ ) are proportional to $\sqrt{g}$ in $f>f_{c}$, while they are proportional to $g$ in $f \ll f_{c}$. As the insert becomes longer, the frequency $f_{c}$ becomes lower [see Eq. (40)], and the frequency range where the radiation effects dominate in the impedance becomes wider.

The physical reason of why the whole wall current tends to run on the thin insert except for the extremely thin insert case is that the nature tries to minimize the energy loss of a beam, which is smaller when the wall current runs on the thin insert with large resistance than when it converts to the radiation out to free space (= gap impedance). When $t \ll$ $t_{\min }$, the real part of the correct impedances using the present theory is smaller than the hypothetical impedances calculated by extending the simple formula (39) to these extreme thicknesses. The real part of the impedance, i.e. the energy loss of a beam, becomes smaller by the wall current converting to outer radiation than it staying in the extremely thin insert.

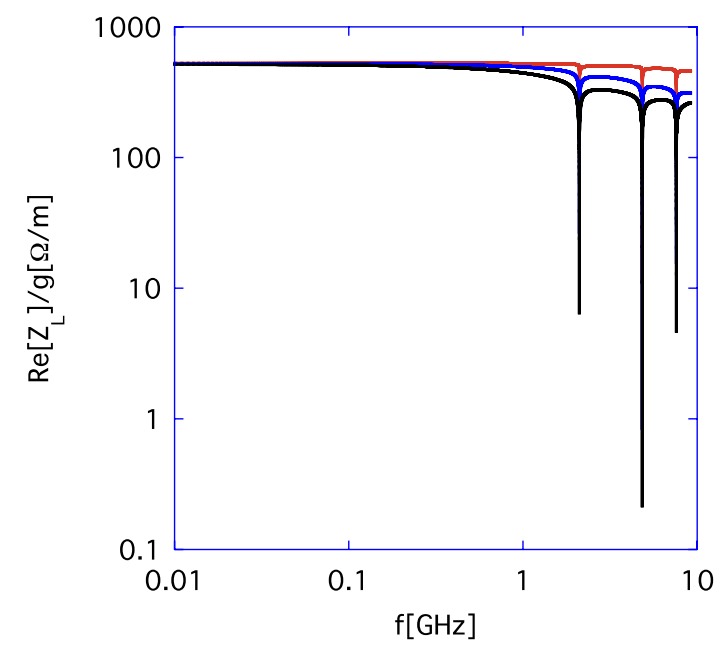

FIG. 5. (Color) The longitudinal impedances per a unit length for the case that the thickness of the insert $t$ is equal to $1 \mathrm{~nm}$ and Lorentz factor $\gamma$ is equal to 1000 . The red, the blue, and the black lines show the cases that the length of the insert $g$ is equal to 2,8 , and $18 \mathrm{~mm}$, respectively. The impedance itself (not normalized by the gap length $g$ ) is proportional to $\sqrt{g}$ in $f>f_{c}$, while it is proportional to $g$ in $f \ll f_{c}$. 
Finally, we briefly mention the nonrelativistic beam case for the insert that satisfies $t>t_{\min }$ as a typical case. The same discussion as in the relativistic beam case is valid in this case as well. Simple formula which is based on the formulas

$$
Z_{\mathrm{wall}}=\frac{g}{\pi \sigma_{c}\left[(a+\delta)^{2}-a^{2}\right]},
$$

and Eq. (39) in the frequency region where the skin depth is smaller than the thickness $t$, and is larger than the thickness $t$, respectively, provides good approximation to the correct impedance of the insert.

\section{TRANSVERSE IMPEDANCE OF A RESISTIVE INSERT IN A BEAM PIPE}

Following the manipulation for the longitudinal case, we consider the situation where a beam is traveling in the chamber with the charge distribution of the azimuthal dependence as $j_{z}=q \beta c \delta\left(\rho-r_{b}\right) \cos \theta e^{-j k z} / \pi r_{b}$. At first, we describe fields inside the chamber as in Ref. [12] (see Appendix A):

$$
\begin{aligned}
E_{z}= & i_{1}\left[\frac{j k}{\gamma^{2}} \frac{c Z_{0}}{\pi}\left(K_{1}\left(\bar{k} r_{b}\right)-K_{1}(\bar{k} a) \frac{I_{1}\left(\bar{k} r_{b}\right)}{I_{1}(\bar{k} a)}\right) I_{1}(\bar{k} \rho) \frac{e^{-j k z}}{r_{b}}\right. \\
& \left.+\tilde{V}_{2} \int_{-\infty}^{\infty} d h e^{-j h z} \frac{\sin h w}{h w} \frac{J_{1}(\Lambda \rho)}{J_{1}(\Lambda a)}\right] \cos \theta
\end{aligned}
$$

for $\rho<r_{b}$ and

$$
\begin{gathered}
E_{z}=i_{1}\left[\frac{j k}{\gamma^{2}} \frac{c Z_{0}}{\pi} K_{1}(\bar{k} \rho) I_{1}\left(\bar{k} r_{b}\right) \frac{e^{-j k z}}{r_{b}}\right. \\
\left.+\int_{-\infty}^{\infty} d h A(h) e^{-j h z} \frac{J_{1}(\Lambda \rho)}{J_{1}(\Lambda a)}\right] \cos \theta \\
H_{\theta}=i_{1}\left[c \beta \frac{\bar{k}}{2 \pi}\left[K_{0}(\bar{k} \rho)+K_{2}(\bar{k} \rho)\right] I_{1}\left(\bar{k} r_{b}\right) \frac{e^{-j k z}}{r_{b}}\right. \\
-\int_{-\infty}^{\infty} d h e^{-j h z} \frac{j h}{\Lambda^{2}}\left(\frac{B(h) J_{1}(\Lambda \rho)}{\rho J_{1}(\Lambda a)}\right. \\
\left.\left.+\frac{k \beta \Lambda}{Z_{0} h} A(h) \frac{J_{1}^{\prime}(\Lambda \rho)}{J_{1}(\Lambda a)}\right)\right] \cos \theta, \\
H_{z}=i_{1} \int_{-\infty}^{\infty} d h B(h) e^{-j h z} \frac{J_{1}(\Lambda \rho)}{J_{1}(\Lambda a)} \sin \theta, \\
E_{\theta}=i_{1}\left[\frac{c Z_{0}}{\pi \rho} K_{1}(\bar{k} \rho) I_{1}\left(\bar{k} r_{b}\right) \frac{e^{-j k z}}{r_{b}}+\int_{-\infty}^{\infty} d h e^{-j h z} \frac{j k \beta Z_{0}}{\Lambda^{2}}\right. \\
\left.\times\left(B(h) \Lambda \frac{J_{1}^{\prime}(\Lambda \rho)}{J_{1}(\Lambda a)}+\frac{h}{Z_{0} k \beta \rho} A(h) \frac{J_{1}(\Lambda \rho)}{J_{1}(\Lambda a)}\right)\right] \sin \theta,
\end{gathered}
$$

for $\rho>r_{b}$, where $i_{1}=q r_{b}$, and $A(h)$ and $B(h)$ are expan- sion coefficients. Since $E_{z}$ and $E_{\theta}$ on the inner surface of the chamber should be zero except in the insert, the expansion coefficients $A(h)$ and $B(h)$ should satisfy the following relations:

$$
\begin{aligned}
& \frac{j k}{\gamma^{2}} \frac{c Z_{0}}{\pi} K_{1}(\bar{k} a) I_{1}\left(\bar{k} r_{b}\right) \frac{e^{-j k z}}{r_{b}}+\int_{-\infty}^{\infty} d h A(h) e^{-j h z} \\
& \quad= \begin{cases}\frac{\pi \tilde{V}_{2}}{w} & \text { for }-w<z<w \\
0 & \text { otherwise }\end{cases}
\end{aligned}
$$

$$
\begin{aligned}
& \frac{c Z_{0}}{\pi a} K_{1}(\bar{k} \rho) I_{1}\left(\bar{k} r_{b}\right) \frac{e^{-j k z}}{r_{b}} \\
& \quad+\int_{-\infty}^{\infty} d h e^{-j h z} \frac{j k \beta Z_{0}}{\Lambda^{2}}\left(B(h) \Lambda \frac{J_{1}^{\prime}(\Lambda a)}{J_{1}(\Lambda a)}+\frac{h}{Z_{0} k \beta a} A(h)\right) \\
& = \begin{cases}\frac{\pi \tilde{V}_{1}}{w} & \text { for }-w<z<w \\
0 & \text { otherwise, }\end{cases}
\end{aligned}
$$

where $\tilde{V}_{1}$ and $\tilde{V}_{2}$ are the voltages inside the insert at $\rho=a$. Then, the expansion coefficients $A(h)$ and $B(h)$ are rewritten by the insert voltages $\tilde{V}_{1}$ and $\tilde{V}_{2}$ as

$$
\frac{j k}{\gamma^{2}} \frac{c Z_{0}}{\pi} K_{1}(\bar{k} a) I_{1}\left(\bar{k} r_{b}\right) \frac{\delta(h-k)}{r_{b}}+A(h)=\tilde{V}_{2} \frac{\sin h w}{h w},
$$

$$
\begin{aligned}
& \frac{c Z_{0}}{\pi a} K_{1}(\bar{k} \rho) I_{1}\left(\bar{k} r_{b}\right) \frac{\delta(h-k)}{r_{b}} \\
& \quad+\frac{j k \beta Z_{0}}{\Lambda^{2}}\left(B(h) \Lambda \frac{J_{1}^{\prime}(\Lambda a)}{J_{1}(\Lambda a)}+\frac{h}{Z_{0} k \beta a} A(h)\right)=\tilde{V}_{1} \frac{\sin h w}{h w} .
\end{aligned}
$$

Substituting Eqs. (49) and (50) into Eqs. (43)-(46), we obtain

$$
\begin{aligned}
E_{z}=i_{1}\left[\frac{j k}{\gamma^{2}} \frac{c Z_{0}}{\pi}\left(K_{1}(\bar{k} \rho)-\frac{I_{1}(\bar{k} \rho)}{I_{1}(\bar{k} a)} K_{1}(\bar{k} a)\right) I_{1}\left(\bar{k} r_{b}\right) \frac{e^{-j k z}}{r_{b}}\right. \\
\left.+\tilde{V}_{2} \int_{-\infty}^{\infty} d h e^{-j h z} \frac{\sin h w}{h w} \frac{J_{1}(\Lambda \rho)}{J_{1}(\Lambda a)}\right] \cos \theta \\
H_{\theta}=i_{1}\left\{c \beta \frac{\bar{k}}{2 \pi}\left[K_{0}(\bar{k} \rho)+K_{2}(\bar{k} \rho)\right] I_{1}\left(\bar{k} r_{b}\right) \frac{e^{-j k z}}{r_{b}}\right. \\
+c \beta \frac{\bar{k}}{\pi I_{1}(\bar{k} a)} K_{1}(\bar{k} a) I_{1}^{\prime}(\bar{k} \rho) I_{1}\left(\bar{k} r_{b}\right) \frac{e^{-j k z}}{r_{b}} \\
\quad-i_{1} \int_{-\infty}^{\infty} d h e^{-j h z} \frac{j h}{Z_{0} \Lambda}\left[\frac{\tilde{V}_{1} J_{1}(\Lambda \rho)}{j k \beta \rho J_{1}^{\prime}(\Lambda a)}\right. \\
\left.\left.+\tilde{V}_{2}\left(\frac{k \beta J_{1}^{\prime}(\Lambda \rho)}{h J_{1}(\Lambda a)}-\frac{h J_{1}(\Lambda \rho)}{\rho k \beta a \Lambda^{2} J_{1}^{\prime}(\Lambda a)}\right)\right]\right\} \cos \theta
\end{aligned}
$$




$$
\begin{aligned}
H_{z}= & i_{1} \int_{-\infty}^{\infty} d h e^{-j h z} \frac{\sin h w}{h w}\left(\tilde{V}_{1}-\frac{j h \tilde{V}_{2}}{\Lambda^{2} a}\right) \frac{\Lambda}{j k \beta Z_{0}} \frac{J_{1}(\Lambda \rho)}{J_{1}^{\prime}(\Lambda a)} \\
& \times \sin \theta, \\
E_{\theta}= & i_{1}\left[\frac{c Z_{0}}{\pi \rho}\left(K_{1}(\bar{k} \rho)-\frac{I_{1}(\bar{k} \rho)}{I_{1}(\bar{k} a)} K_{1}(\bar{k} a)\right) I_{1}\left(\bar{k} r_{b}\right) \frac{e^{-j k z}}{r_{b}}\right. \\
& +\tilde{V}_{1} \int_{-\infty}^{\infty} d h e^{-j h z} \frac{\sin h w}{h w} \frac{J_{1}^{\prime}(\Lambda \rho)}{J_{1}^{\prime}(\Lambda a)} \\
& \left.+\tilde{V}_{2} \int_{-\infty}^{\infty} d h e^{-j h z} \frac{j h}{\Lambda^{2}} \frac{\sin h w}{h w}\left(\frac{J_{1}(\Lambda \rho)}{\rho J_{1}(\Lambda a)}-\frac{J_{1}^{\prime}(\Lambda \rho)}{a J_{1}^{\prime}(\Lambda a)}\right)\right] \\
& \times \sin \theta,
\end{aligned}
$$

for $\rho>r_{b}$.

Second, we have to know fields outside the chamber to apply the field matching technique [6]. They are (see Appendix B)

$$
\begin{aligned}
E_{z}= & i_{1} V_{2} \int_{-\infty}^{\infty} d h \frac{H_{1}^{(2)}(\Lambda \rho)}{H_{1}^{(2)}[\Lambda(a+t)]} \frac{\sin h w}{h w} e^{-j h z} \cos \theta \\
H_{\theta}= & i_{1} V_{2} \int_{-\infty}^{\infty} d h\left(-j \frac{k \beta}{Z_{0}} \frac{H_{1}^{\prime(2)}(\Lambda \rho)}{\Lambda H_{1}^{(2)}[\Lambda(a+t)]}+j \frac{h^{2}}{\rho}\right. \\
& \left.\times \frac{H_{1}^{(2)}(\Lambda \rho)}{Z_{0} k \beta(a+t) \Lambda^{3} H_{1}^{\prime(2)}[\Lambda(a+t)]}\right) \\
& \times \frac{\sin h w}{h w} e^{-j h z} \cos \theta-i_{1} V_{1} \int_{-\infty}^{\infty} d h \frac{h}{\rho} \\
& \times \frac{H_{1}^{(2)}(\Lambda \rho)}{Z_{0} k \beta \Lambda H_{1}^{\prime(2)}[\Lambda(a+t)]} \frac{\sin h w}{h w} e^{-j h z} \cos \theta \\
H_{z}= & -\int_{-\infty}^{\infty} d h j \Lambda^{2}\left(i_{1} V_{1}-j \frac{i_{1} V_{2} h}{(a+t) \Lambda^{2}}\right) \\
& \times \frac{H_{1}^{(2)}(\Lambda \rho)}{Z_{0} k \beta \Lambda H_{1}^{\prime(2)}[\Lambda(a+t)]} \frac{\sin h w}{h w} e^{-j h z} \sin \theta
\end{aligned}
$$

$$
\begin{aligned}
E_{\theta}= & i_{1} V_{2} \int_{-\infty}^{\infty} d h\left(j \frac{h}{\rho \Lambda^{2}} \frac{H_{1}^{(2)}(\Lambda \rho)}{H_{1}^{(2)}[\Lambda(a+t)]}-j \frac{h}{(a+t) \Lambda^{2}}\right. \\
& \left.\times \frac{H_{1}^{\prime(2)}(\Lambda \rho)}{H_{1}^{\prime(2)}[\Lambda(a+t)]}\right) \frac{\sin h w}{h w} e^{-j h z} \sin \theta \\
& +i_{1} V_{1} \int_{-\infty}^{\infty} d h \frac{H_{1}^{\prime(2)}(\Lambda \rho)}{H_{1}^{\prime(2)}[\Lambda(a+t)]} \frac{\sin h w}{h w} e^{-j h z} \sin \theta,
\end{aligned}
$$

where $H_{m}^{(2)}(z)$ is the Hankel function of the second kind, the prime means the differential by its argument $z, V_{1}$, and $V_{2}$ are the voltages inside the insert at $\rho=a+t$. In the process of obtaining the above equation, we used the approximation that $V_{1}$ and $V_{2}$ are almost constant.

We have to find the relation between fields at $\rho=a$ and those at $\rho=a+t$. Similarly to the longitudinal impedance, let us assume that the relation is approximately obtained by solving the one-dimensional problem as shown in Fig. 2. Maxwell equations in region II depicted in Fig. 2 are given by

$$
\begin{gathered}
\frac{\partial E_{\theta}}{\partial x}(x)=-j k \beta Z_{0} H_{z}(x), \\
-\frac{\partial H_{z}(x)}{\partial x}=\left(\sigma_{c}+j \frac{k \beta \epsilon^{\prime}}{Z_{0}}\right) E_{\theta}(x),
\end{gathered}
$$

in addition to Eqs. (14) and (15). In addition to Eqs. (16) and (17), the solutions are

$$
\begin{aligned}
E_{\theta}(x)= & E_{\theta}(0) \cosh \sqrt{j k \beta Z_{0}\left(\sigma_{c}+j \frac{k \beta \epsilon^{\prime}}{Z_{0}}\right) x} \\
& -H_{z}(0) \frac{j Z_{0} k \beta \sinh \sqrt{j k \beta Z_{0}\left(\sigma_{c}+j \frac{k \beta \epsilon^{\prime}}{Z_{0}}\right)} x}{\sqrt{j k \beta Z_{0}\left(\sigma_{c}+j \frac{k \beta \epsilon^{\prime}}{Z_{0}}\right)}},
\end{aligned}
$$

Since the fields given by Eqs. (51)-(54) and those given by Eqs. (55)-(58) must be connected through these relations [Eqs. (16), (17), (61), and (62)], one of the insert voltages $\tilde{V}_{2}$ can be solved as

$$
\tilde{V}_{2}=\frac{j c Z_{0} I_{1}\left(\bar{k} r_{b}\right) e^{-j k z}}{\pi k a r_{b} I_{1}(\bar{k} a)\left(-\frac{\pi \sqrt{j k \beta Z_{0} \sigma_{c}}}{k^{2} \beta^{2} w} \tanh \sqrt{j k \beta Z_{0} \sigma_{c}} t+P-P_{3}\right)},
$$




$$
\begin{gathered}
P=\frac{1}{2 w} \int_{-\infty}^{\infty} d h \int_{-w}^{w} d \xi \frac{e^{-j h(z-\xi)}}{\Lambda}\left(\frac{H_{1}^{\prime(2)}[\Lambda(a+t)]}{H_{1}^{(2)}[\Lambda(a+t)]}-\frac{J_{1}^{\prime}(\Lambda a)}{J_{1}(\Lambda a)}\right), \\
P_{3}=\frac{1}{2 w} \int_{-\infty}^{\infty} d h \int_{-w}^{w} d \xi e^{-j h(z-\xi)} \frac{h^{2}}{k^{2} \beta^{2} \Lambda^{3}}\left(\frac{H_{1}^{(2)}(\Lambda(a+t))}{(a+t)^{2} H_{1}^{\prime(2)}[\Lambda(a+t)]}-\frac{J_{1}(\Lambda a)}{a^{2} J_{1}^{\prime}(\Lambda a)}\right),
\end{gathered}
$$

where $z$ is the matching point of magnetic fields.

If we substitute Eq. (63) into Eq. (42) and use the Panofsky-Wenzel theorem [7,16], we can finally obtain the expression for the transverse impedance as

$$
Z_{T}=\frac{R Z_{0} k}{j \gamma^{3} \beta r_{b}}\left(K_{1}\left(\bar{k} r_{b}\right)-K_{1}(\bar{k} a) \frac{I_{1}\left(\bar{k} r_{b}\right)}{I_{1}(\bar{k} a)}\right)-\frac{\pi \tilde{V}_{2}}{c \beta \gamma I_{1}(\bar{k} a)} \frac{\sin k w}{k w} .
$$

The first term of Eq. (66) represents the transverse space charge impedance for a nonrelativistic beam (see Appendix A) [12], while the second term gives the transverse insert impedance $Z_{T \text {,insert }}$. Using Eqs. (C1), (C2), (C4), and (C5), we can rewrite $Z_{T \text {,insert }}$ as

$$
Z_{T, \text { insert }} \simeq-\frac{j Z_{0} I_{1}\left(\bar{k} r_{b}\right) e^{-j k z}}{\beta \gamma r_{b} a k I_{1}^{2}(\bar{k} a)\left(-\frac{\pi \sqrt{j k \beta Z_{0} \sigma_{c}}}{k^{2} \beta^{2} w} \tanh \sqrt{j k \beta Z_{0} \sigma_{c}} t+Y_{\text {pole }}^{\prime}+Y_{\text {cut }}^{\prime}\right)},
$$

where

$$
\begin{aligned}
Y_{\mathrm{pole}}^{\prime}= & \sum_{s=1}^{\infty}\left[-\frac{\pi a\left(2-e^{-j\left(b_{1, s} / a\right)(z+w)}-e^{j\left(b_{1, s} / a\right)(z-w)}\right)}{w b_{1, s}^{2}}+\frac{\pi a J_{1}\left(j_{1, s}^{\prime}\right)\left(2-e^{-j\left(b_{1, s}^{\prime} / a\right)(z+w)}-e^{j\left(b_{1, s}^{\prime} / a\right)(z-w)}\right)}{k^{2} \beta^{2} a^{2} w j_{1, s}^{\prime 2} J_{1}^{\prime \prime}\left(j_{1, s}^{\prime}\right)}\right] \\
& -\frac{\pi H_{1}^{(2)}\left(h_{1,0}^{\prime}\right)\left(2-e^{-j\left[d_{1,0}^{\prime} /(a+t)\right](z+w)}-e^{j\left[d_{1,0}^{\prime} /(a+t)\right](z-w)}\right)}{k^{2} \beta^{2}(a+t) w h_{1,0}^{\prime 2} H_{1}^{\prime \prime(2)}\left(h_{1,0}^{\prime}\right)}+\frac{\pi\left(2-e^{-j k \beta(z+w)}-e^{j k \beta(z-w)}\right)}{w k^{2} \beta^{2}} \\
& \times\left(\frac{H_{1}^{(2)}\left(h_{1,0}^{\prime}\right)}{(a+t) h_{1,0}^{\prime 2} H_{1}^{\prime \prime(2)}\left(h_{1,0}^{\prime}\right)}-\frac{1}{2 a}\right), \\
& Y_{\text {cut }}^{\prime}=-\frac{1}{\pi(a+t) w} \int_{0}^{\infty} d \zeta \frac{\left(2-e^{-j(z+w) \sqrt{k^{2} \beta^{2}+\zeta /(a+t)^{2}}}-e^{j(z-w) \sqrt{k^{2} \beta^{2}+\zeta /(a+t)^{2}}}\right)}{\zeta\left(k^{2} \beta^{2}+\frac{\zeta}{(a+t)^{2}}\right) H_{1}^{(1)}\left(e^{j(\pi / 2)} \sqrt{\zeta}\right) H_{1}^{(2)}\left(e^{j(\pi / 2)} \sqrt{\zeta}\right)} \\
& +\int_{0}^{\infty} d \zeta \frac{\left(e^{-j(z+w) k \beta}+e^{j(z-w) k \beta}-e^{-j(z+w) \sqrt{k^{2} \beta^{2}+\zeta /(a+t)^{2}}}-e^{j(z-w) \sqrt{k^{2} \beta^{2}+\zeta /(a+t)^{2}}}\right)}{k^{2} \beta^{2}(a+t) \pi w \zeta^{2} H_{1}^{\prime(1)}\left(e^{j(\pi / 2)} \sqrt{\zeta}\right) H_{1}^{\prime(2)}\left(e^{j(\pi / 2)} \sqrt{\zeta}\right)} \\
& \simeq 4 \tan ^{-1} \frac{1}{\sqrt{2 j k w}}+\frac{-2+4 \sqrt{1+2 j k \beta w} \sinh ^{-1} \frac{e^{-j(\pi / 4)}}{\sqrt{2 k \beta w}}+e^{-j(z+w) k \beta}+e^{j(z-w) k \beta}}{k^{2} \beta^{2}(a+t)^{2} \sqrt{1+2 j k \beta w}},
\end{aligned}
$$

where $\quad b_{1, s}=\sqrt{k^{2} \beta^{2} a^{2}-j_{1, s}^{2}}, \quad b_{1, s}^{\prime}=\sqrt{k^{2} \beta^{2} a^{2}-j_{1, s}^{\prime 2}}$, $d_{1,0}^{\prime}=\sqrt{k^{2} \beta^{2} a^{2}-h_{1,0}^{\prime 2}}, j_{n, s}$ are the sth zeros of $J_{n}(z), j_{1, s}^{\prime}$ are the sth zeros of $J_{1}^{\prime}(z), h_{1,0}^{\prime}=0.501184+j 0.643545$ : the 0th zero of $H_{1}^{\prime(2)}(z)$ (the differential of the Hankel function of the second kind) and $H_{1}^{(1)}(z)$ is the Hankel function of the first kind. We should notice that $b_{1, s}^{\prime}$ approaches $-j \sqrt{j_{1, s}^{\prime 2}-k^{2} \beta^{2} a^{2}}$ for $j_{1, s}^{\prime 2}>k^{2} \beta^{2} a^{2}$. Again, we reach the equation where the integration over $\zeta$ is just straightforward for numerical calculations, since there is no singular point along the integration path.

\section{A. Parameter dependence of the transverse impedance}

Now let us examine the frequency dependence of the transverse impedance. We start to study from the extremely high frequency and then will gradually lower the frequency. When the thickness of the insert is larger than $2^{1 / 2} \pi^{3 / 4} t_{\min }$ [see Eq. (26)], the wakefield leaks out of the 
insert in the high frequency region specified by $f \gg f_{D}$ [see Eq. (27)]. In this frequency range, the transverse impedance is approximately given by

$$
Z_{T} \simeq \frac{(1-j) Z_{0} \sqrt{k g}}{8 \sqrt{2} \beta \gamma^{2} a I_{1}^{2}(\bar{k} a)},
$$

which becomes for a relativistic beam

$$
Z_{T} \simeq \frac{(1-j) Z_{0 \sqrt{g}}}{2 \sqrt{2} k^{3 / 2} a^{3}}
$$

In the frequency region where $f \ll f_{D}$ but still the skin depth is smaller than the insert thickness, the impedance is approximately written as

$$
Z_{T, \text { insert }} \simeq \frac{\sqrt{j k \beta Z_{0}} g I_{1}\left(\bar{k} r_{b}\right)}{2 \gamma \pi r_{b} a \sqrt{\sigma_{c}} I_{1}^{2}(\bar{k} a)},
$$

which reproduces the conventional resistive-wall impedance for a relativistic beam [7]:

$$
Z_{T, \text { insert }}=g c \sqrt{\frac{Z_{0} \omega \sigma_{c}}{2 c}} \frac{1+j}{\pi \sigma_{c} \omega a^{3}} .
$$

In a lower frequency where the skin depth exceeds the thickness of the insert, the impedance becomes

$$
Z_{T, \text { insert }}=\frac{g c}{2 \pi^{2} f a^{3} \sigma_{c} t} .
$$

When the thickness of the insert is smaller than $2^{1 / 2} \pi^{3 / 4} t_{\min }$ but larger than $t_{\min }$, Eq. (74) gives correct impedance all the way up to $f_{D}$. Finally, in the region of

$$
f \ll f_{L} \equiv \frac{3 c}{4 \pi Z_{0} \sigma_{c} t a},
$$

the wakefields leak out of the insert again. It is almost identical to the gap impedance $Z_{g a p, \perp}$ described in Ref. [12], and goes down toward zero as the frequency approaches to zero.

Now, we consider the thickness dependence of the insert. The dependence of the real part of the insert impedance on the insert thickness is shown in Fig. 6. The red, the blue, the black, the black dashed, the black dot, the green, the blue dashed, and the red dashed lines represent the cases for the thickness $t$ equal to $100 \mu \mathrm{m}, 10 \mu \mathrm{m}, 1 \mu \mathrm{m}$, $100 \mathrm{~nm}, 10 \mathrm{~nm}, 1 \mathrm{~nm}, 100 \mathrm{pm}$, and $10 \mathrm{pm}$, respectively. Similar to the longitudinal case, we at first consider the case that the thickness of the insert $t$ is larger than $t_{\min }$. The result of $t=100 \mu \mathrm{m}$ in Fig. 6 corresponds to the case that the skin depth $\delta$ is smaller than the thickness of the insert $t$, which reproduces Eq. (73). The results of $t=10 \mu \mathrm{m}$ to $t=100 \mathrm{~nm}$ in Fig. 6 represent the case that the skin depth $\delta$ exceeds the thickness of the chamber $t$ except at the low frequency extreme $f \ll f_{L}$ [see Eq. (75)]. These impedances (even including the result for $10 \mathrm{~nm}$ which is smaller than $t_{\min }$ ) agree very well with those obtained from the simple formula,

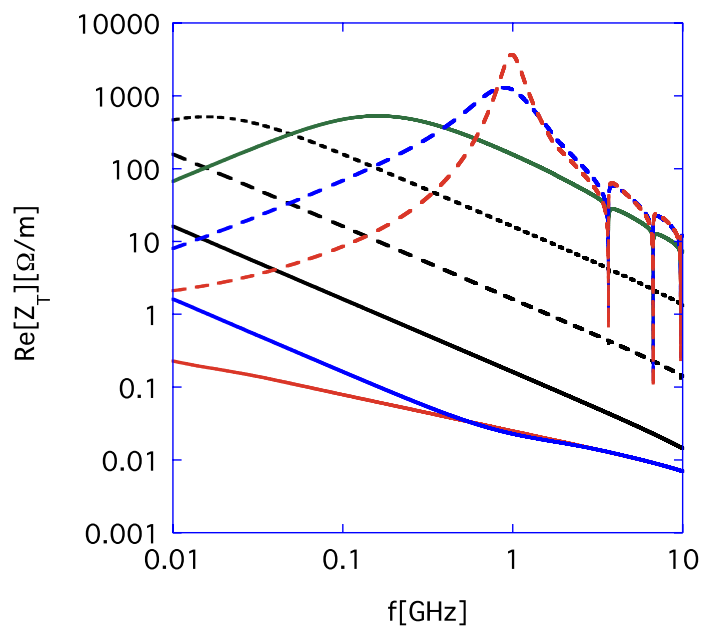

FIG. 6. (Color) The thickness dependence of the transverse impedance of the insert for the relativistic beam case. The red, the blue, the black, the black dashed, the black dot, the green, the blue dashed, and the red dashed lines represent the cases that thickness $t$ is equal to $100 \mu \mathrm{m}, 10 \mu \mathrm{m}, 1 \mu \mathrm{m}, 100 \mathrm{~nm}, 10 \mathrm{~nm}$, $1 \mathrm{~nm}, 100 \mathrm{pm}$, and $10 \mathrm{pm}$, respectively. The impedance becomes proportional to $f^{-1}$, when the skin depth exceeds the insert thickness. The matching point is $z=0$.

$$
\Re\left[Z_{T}\right] \simeq \frac{2 \beta c}{a^{2} \omega} Z_{\text {wall }}=\frac{\beta c g}{2 \pi^{2} f a^{3} \sigma_{c} t},
$$

where $Z_{\text {wall }}$ is identical to Eq. (39). The case of $t=10 \mu \mathrm{m}$ especially helps us to understand the behavior of the real part of the impedance, which starts to deviate from Eq. (73) and becomes proportional to $f^{-1}$ when the skin depth exceeds the insert thickness $\left[f<f_{\delta}\right.$; see Eq. (33)]. In the frequency region specified by $f_{L}<f<f_{\delta}$, the whole wall current runs on the thin insert, and wakefields are still confined inside the chamber. Contrary to the longitudinal impedance, this picture of the insert impedance is applicable to that of the resistive-wall impedance for the transverse impedance. We numerically calculate the resistivewall impedance for different thicknesses of the chamber by borrowing the general formula (A49) of the resistive-wall impedance with finite thickness from the recent work of Metral et al. [10] (see Appendix A 2). The results for a relativistic beam are shown in Fig. 7. The red, the blue, the black, the black dashed, and the black dotted lines show the cases that the insert thickness $t$ is equal to infinity, $10 \mu \mathrm{m}$, $1 \mu \mathrm{m}, 100 \mathrm{~nm}$, and $10 \mathrm{~nm}$, respectively. The entire wall current runs on the chamber for the resistive-wall impedance as well, after the skin depth exceeds the chamber thickness. But at the region $f<f_{L}$ (but not quite lower as in the short insert) where the skin depth is much larger than the chamber thickness, the resistive-wall impedance starts to fall off.

In the case that the thickness of the insert $t$ is extremely thin like $t \ll t_{\min }$, the situation becomes significantly different. The results of $t=1 \mathrm{~nm}$ to $10 \mathrm{pm}$ in Fig. 6 corre- 


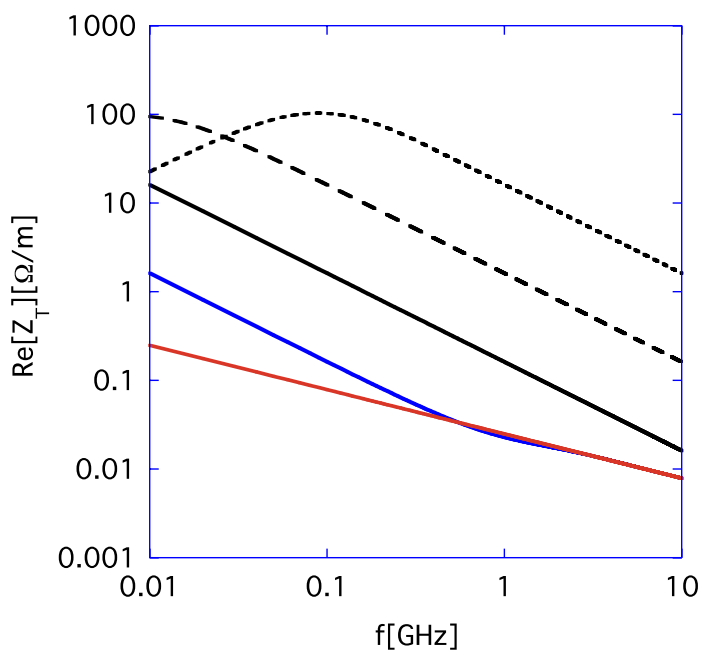

FIG. 7. (Color) The dependence of the real part of the transverse resistive-wall impedance of a uniform beam pipe (no insert) on the thickness of the chamber. The length of the beam pipe $g=$ $8 \mathrm{~mm}$. Lorentz factor $\gamma=1000$. The red, the blue, the black, the black dashed, and the black dotted lines show the cases that the chamber thickness $t$ is equal to infinity, $10 \mu \mathrm{m}, 1 \mu \mathrm{m}, 100 \mathrm{~nm}$, and $10 \mathrm{~nm}$, respectively. The impedance for finite thickness starts to deviate from that for infinite thickness at the frequency where the skin depth exceeds the chamber thickness.

spond to this case. The parameter $f_{L}$ should be replaced by a new parameter:

$$
f_{r} \equiv \frac{1}{2 \pi}\left(\frac{2 g c^{3}}{Z_{0}^{2} \sigma_{c}^{2} a^{4} t^{2}}\right)^{1 / 3} .
$$

Contrary to the longitudinal case, $f_{r}$ as well as $f_{c}$ [see Eq. (40)] are used to classify the property of the impedance along the frequency axis. In the frequency region $f_{r}<$ $f \ll f_{c}$, the contribution from the wall current dominates in the impedance, while the radiation effects dominate in the rest of the frequency. Figure 8 shows the dependence of the impedance on the length of the insert $g$ where the thickness of the insert $t$ is equal to $10 \mathrm{~nm}$ and Lorentz factor $\gamma$ is equal to 1000 . The red, the blue, and the black lines show the transverse impedance per a unit length for the cases that the length of the insert $g$ is equal to $2 \mathrm{~mm}$, $8 \mathrm{~mm}$, and $18 \mathrm{~mm}$, respectively. Since the wall current effect dominates in the impedance in the frequency region $f_{r}<f \ll f_{c}$, the impedance is proportional to the length of the insert $g$. The impedance is proportional to $\sqrt{g}$ in the higher frequency region, as the contributions from the radiation dominate in the impedance. The wakefield makes dips in the impedance curve for the frequency that is larger than the cutoff frequency of the chamber. Especially, in the case of the infinitesimally thin insert, the impedance is identical to the gap impedance, in the entire frequency. Like the longitudinal case, the transition thickness of the insert at which the wall current starts converting to the outer radiation from running on the thin insert is deter-

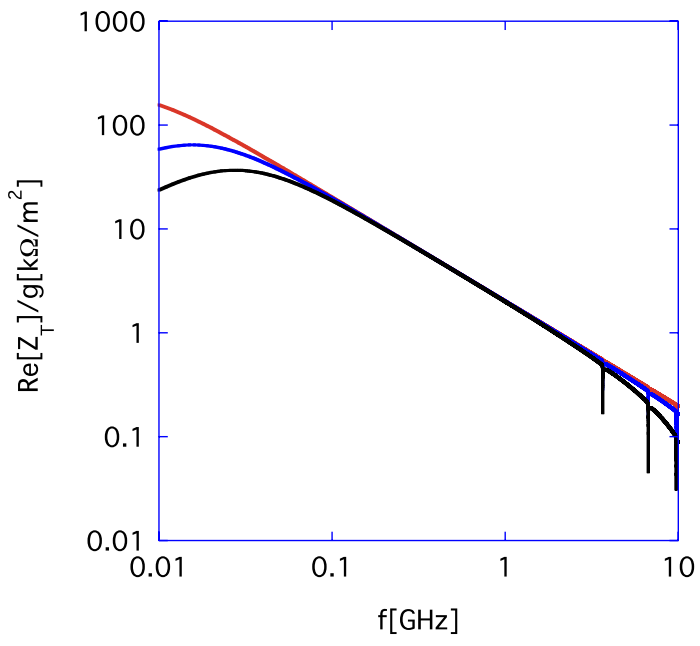

FIG. 8. (Color) The real part of the transverse impedance per a unit length for the case that the thickness of the insert $t$ is equal to $10 \mathrm{~nm}$ and Lorentz factor $\gamma$ is equal to 1000 . The red, the blue, and the black lines show the case the length of the insert $g$ is equal to 2,8 , and $18 \mathrm{~mm}$, respectively.

mined by which case minimizes the impedance and thus the energy loss of a beam. Figure 9 demonstrates this fact by comparing the correct impedances with the hypothetical ones obtained by extending the simple formula (76) to these extreme thicknesses.

Finally we briefly mention the nonrelativistic beam case. The same discussion as in the relativistic beam case is valid in this case as well. The transverse resistive-wall impedance $[10,11]$ well describes that of a short insert except the extremely thin case like $t<t_{\min }$ and in the low frequency extreme: $f \ll f_{L}$.

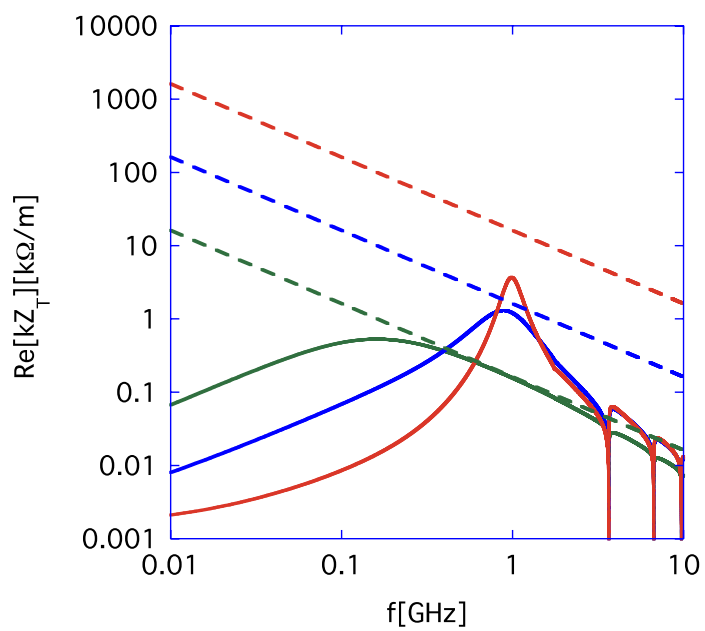

FIG. 9. (Color) The transverse impedances for the case of $t \ll$ $t_{\min }$. The green solid and dashed, the blue solid and dashed, and the red solid and dashed lines show the cases that the thickness of the insert $t$ is equal to $1 \mathrm{~nm}, 100 \mathrm{pm}$, and $10 \mathrm{pm}$, respectively. The solid lines are based on the present theory, while the dashed lines are calculated hypothetically by extending the simple formula (76) to these extreme thicknesses. 


\section{SUMMARY}

We have developed the theory to describe the longitudinal and the transverse impedances of a short insert with cylindrical symmetry sandwiched by the perfectly conductive chambers by generalizing the theory of a gap impedance. When the thickness of the insert $t$ is larger than $2^{1 / 2} \pi^{3 / 4} t_{\min }\left[=\left(16 g \pi / Z_{0}^{3} \sigma_{c}^{3}\right)^{1 / 4}\right]$, the impedance by our theory in the frequency region of $f_{\delta}\left(=c / \pi Z_{0} \sigma_{c} t^{2}\right)<$ $f \ll f_{D}\left(=\sqrt{c^{2} Z_{0} \sigma_{c} / 2 \pi^{2} g}\right)$ reproduces the results of the resistive-wall impedance. Moreover, the result by our theory in the higher frequency region is consistent with the prediction of the diffraction theory. Formulas valid for various cases of inserts (including the resistive-wall impedance) are categorized in Table I.

An interesting finding for the short insert is that when the thickness of the insert $t$ is larger than $t_{\min }[=(4 \mathrm{~g} /$ $\left.\pi^{2} Z_{0}^{3} \sigma_{c}^{3}\right)^{1 / 4}$ ] (typically a few $10 \mathrm{~nm}$ for a metallic insert and a few times larger than the thickness of TiN coating inside a short ceramic break in a proton synchrotron), the entire wall current runs in the thin insert even when the skin depth exceeds the thickness of the insert [strictly speaking, except the low frequency extreme given by $f \ll f_{L}(=3 c /$ $\left.4 \pi Z_{0} \sigma_{c} t a\right)$ for the transverse impedance] and therefore the impedances increase drastically from the conventional resistive-wall impedance. This feature of the short insert does not depend on whether a beam is relativistic or not. In other words, this behavior is quite different from that of the resistive-wall impedances, especially from the longitudinal impedance for a relativistic beam. In this resistive-wall case the wakefields immediately propagate out of the chamber once the skin depth exceeds the thickness of the chamber.

The physical reason of why the whole wall current tends to run on the thin insert except for the extremely thin insert case is that the nature tries to minimize the energy loss of a beam, which is smaller when the wall current runs on the thin insert with large resistance than when it converts to the radiation out to free space (= gap impedance).

Only when the thickness of the insert $t$ is smaller than $t_{\min }$, the contribution from the wall current in the impedance of the short insert starts to diminish. For the longitudinal impedance, the parameter $f_{c}\left(=\sigma_{c}^{2} Z_{0}^{2} t^{2} c / 4 \pi g\right)$ specifies the upper limit of the frequency where the wall current effects are dominant in the impedance. As the insert becomes thinner, this upper limit moves to a lower frequency. For the transverse impedance, the another parameter $f_{r}\left[=\left(g c^{3} / 4 \pi^{3} Z_{0}^{2} \sigma_{c}^{2} a^{4} t^{2}\right)^{1 / 3}\right]$ specifies the lower limit of the frequency region where the wall current effects are dominant. As the insert becomes thinner, the lower limit moves to a higher frequency, and as a result, the frequency region where the wall current effects dominate in the impedance becomes narrower from the both sides. Finally, for the extremely thin insert, both the longitudinal and the transverse impedances by our theory converge to those of the gap impedance.

Since these parameters $f_{c}$ and $f_{r}$, that specify the wall current dominant region, are proportional to $t^{2} / g$ and

TABLE I. Valid formulas categorized according to various cases of inserts, where $a$ is the radius of the chamber, $g$ is the length of the insert, $t$ is the thickness of the insert, $c$ is the velocity of light, $Z_{0}(=120 \pi)$ is the impedance of free space, $\sigma_{c}$ is the conductivity of the insert, $f$ is the frequency, $\delta\left(=\sqrt{c / \pi Z_{0} f \sigma_{c}}\right)$ is the skin depth, $f_{\delta}=c / \pi Z_{0} \sigma_{c} t^{2}, f_{L}=3 c / 4 \pi Z_{0} \sigma_{c} t a, f_{D}=$ $\sqrt{c^{2} Z_{0} \sigma_{c} / 2 \pi^{2} g}, f_{c}=\sigma_{c}^{2} Z_{0}^{2} t^{2} c / 4 \pi g, f_{r}=\left(g c^{3} / 4 \pi^{3} Z_{0}^{2} \sigma_{c}^{2} a^{4} t^{2}\right)^{1 / 3}$, and $t_{\min }=\left(4 g / \pi^{2} Z_{0}^{3} \sigma_{c}^{3}\right)^{1 / 4}$.

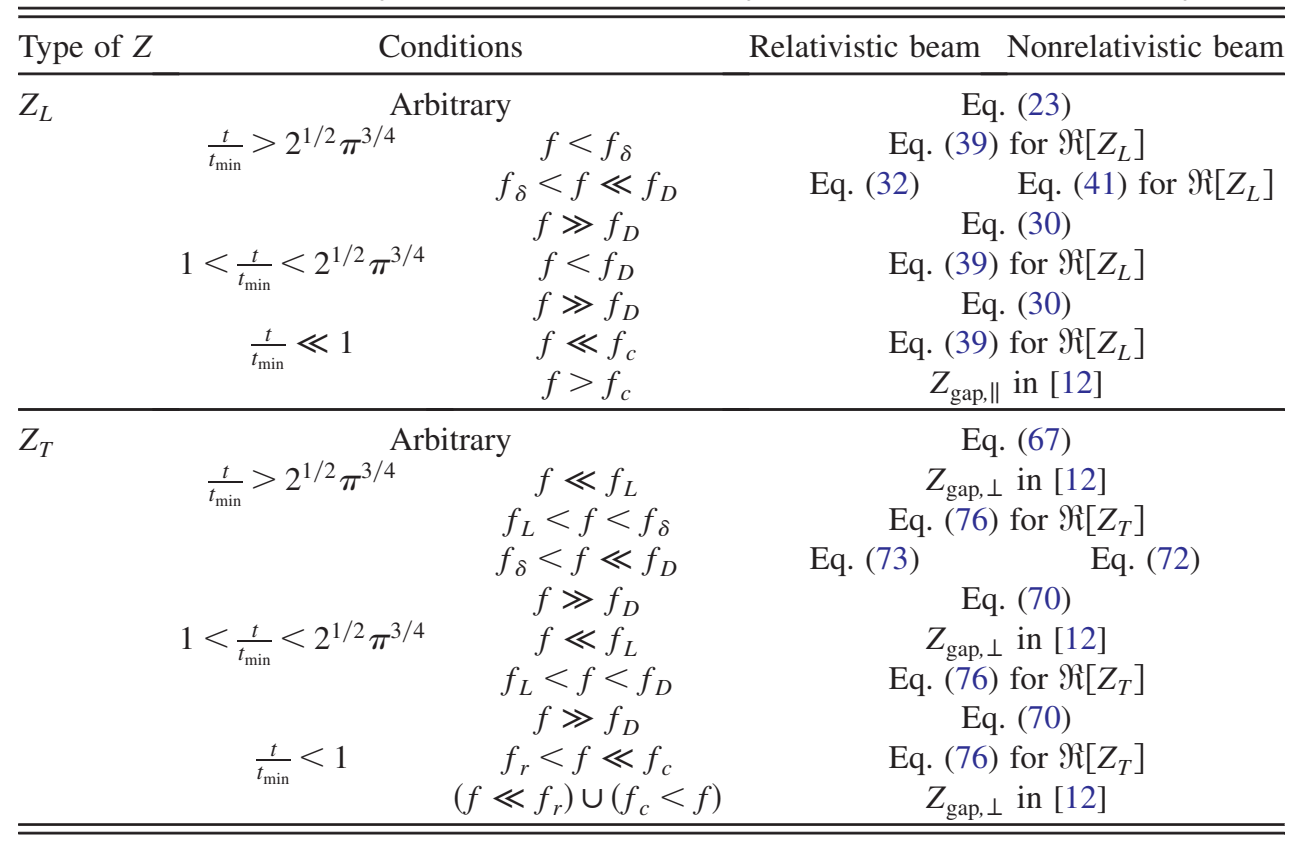


$\left(g / t^{2}\right)^{1 / 3}$, respectively, the increase of the length of the insert $g$ has a similar effect on the impedance as the reduction of the insert thickness, especially when the thickness of the insert is smaller than $t_{\min }$.

It is remarkable that the parameters $t_{\min }$ and $f_{D}$ are proportional to $g^{1 / 4}$ and $g^{-1 / 2}$, respectively. Then, the longer the length of the insert $g$ is, the more easily the radiation effect appears in the impedances. In general, as the insert becomes longer (but still shorter than the radius of the chamber), the wakefields tend to propagate out of the insert.

\section{ACKNOWLEDGMENTS}

The authors would like to thank E. Metral, B. Zotter, and F. Caspers for discussions. The authors would like to express special thanks to E. Metral for his hospitality and help during their stay at CERN. The authors also would like to thank the members of J-PARC project at JAEA/ KEK.

\section{APPENDIX A: SOLUTIONS INSIDE THE CHAMBER}

The Maxwell equations can be written as wave equations. Assuming that electromagnetic fields have time dependency of $e^{j \omega t}$, they become Helmholtz equations:

$$
\begin{gathered}
\left(\Delta+k^{2} \beta^{2}\right) \vec{E}=j k \beta Z_{0} \vec{j}+\vec{\nabla}\left(c Z_{0} \bar{\rho}\right), \\
\left(\Delta+k^{2} \beta^{2}\right) \vec{H}=-\vec{\nabla} \times \vec{j} .
\end{gathered}
$$

In the cylindrical coordinates for an axially symmetric structure, the wave equation for the longitudinal component of the electric and magnetic field contains no transverse field component. They are decoupled. For the longitudinal field, there is a source term $c Z_{0} \partial \bar{\rho} / \partial z+$ $j k \beta Z_{0} j_{z}$, while the $z$ component of $\vec{\nabla} \times \vec{j}$ vanishes for particles with the longitudinal velocity only.

We assume that a macroparticle with charge $q$ travels along the pipe at the constant radial offset position $\rho=r_{b}$, $\theta=\theta_{b}$ with velocity $\beta c$ in the longitudinal direction.
Then, the charge density is expressed as

$$
\begin{gathered}
\bar{\rho}=\frac{i_{m}}{r_{b}^{1+m}} \delta\left(\rho-r_{b}\right) \delta_{p}\left(\theta-\theta_{b}\right) \delta(z-\beta c t) \\
=\sum_{m=0}^{\infty} \int \frac{d k}{2 \pi} i_{m} \rho_{m}, \\
\rho_{m}=\frac{1}{\pi r_{b}^{1+m}\left(1+\delta_{m 0}\right)} \delta\left(\rho-r_{b}\right) \cos m\left(\theta-\theta_{b}\right) e^{-j k(z-\beta c t)}, \\
i_{m}=q r_{b}^{m},
\end{gathered}
$$

where $\delta(x)$ is the $\delta$ function, $\delta_{p}(\theta)$ is the periodic $\delta$ function, and $\delta_{m, n}$ is the Kronecker $\delta$. Since the general solution of Maxwell equations is obtained by the superposition of those for $i_{m} \rho_{m}$, we choose $i_{m} \rho_{m}$ as the source term. Let us define the source field specified with subscript $S$ as the solution which satisfies the Maxwell equations with $\rho_{m}, \vec{j}_{m}$ and vanishes at $\rho \rightarrow \infty$. It is given by

$$
H_{\rho}^{S}=E_{\theta}^{S}=H_{z}^{S}=0
$$

$$
E_{z}^{S}= \begin{cases}\frac{j k c Z_{0} I_{0}\left(\bar{k} r_{b}\right)}{2 \pi \gamma^{2}} K_{0}(\bar{k} \rho) e^{-j k z} & \text { for } \rho>r_{b}, \\ \frac{j k c Z_{0} K_{0}\left(\bar{k} r_{b}\right)}{2 \pi \gamma^{2}} I_{0}(\bar{k} \rho) e^{-j k z} & \text { for } r_{b}>\rho,\end{cases}
$$

$$
\frac{\beta}{Z_{0}} E_{\rho}^{S}=H_{\theta}^{S}= \begin{cases}\frac{\beta k c I_{0}\left(\bar{k} r_{b}\right)}{2 \pi \gamma} K_{1}(\bar{k} \rho) e^{-j k z} & \text { for } \rho>r_{b} \\ -\frac{\beta k c K_{0}\left(\bar{k} r_{b}\right)}{2 \pi \gamma} I_{1}(\bar{k} \rho) e^{-j k z} & \text { for } r_{b}>\rho\end{cases}
$$

for $m=0$, and

$$
H_{z}^{S}=0
$$

$$
E_{z}^{S}= \begin{cases}\frac{j k c Z_{0} I_{m}\left(\bar{k} r_{b}\right)}{\pi r_{b}^{m} \gamma^{2}} K_{m}(\bar{k} \rho) \cos m\left(\theta-\theta_{b}\right) e^{-j k z} & \text { for } \rho>r_{b}, \\ \frac{j k c Z_{0} K_{m}\left(\bar{k} r_{b}\right)}{\pi r_{b}^{m} \gamma^{2}} I_{m}(\bar{k} \rho) \cos m\left(\theta-\theta_{b}\right) e^{-j k z} & \text { for } r_{b}>\rho,\end{cases}
$$

$$
\begin{gathered}
-\frac{Z_{0}}{\beta} H_{\rho}^{S}=E_{\theta}^{S}= \begin{cases}\frac{m c Z_{0} I_{m}\left(\bar{k} r_{b}\right)}{\rho \pi r_{b}^{m}} K_{m}(\bar{k} \rho) \sin m\left(\theta-\theta_{b}\right) e^{-j k z} & \text { for } \rho>r_{b}, \\
\frac{m c Z_{0} K_{m}\left(\bar{k} r_{b}\right)}{\rho \pi r_{b}^{m}} I_{m}(\bar{k} \rho) \sin m\left(\theta-\theta_{b}\right) e^{-j k z} & \text { for } r_{b}>\rho,\end{cases} \\
\frac{\beta}{Z_{0}} E_{\rho}^{S}=H_{\theta}^{S}= \begin{cases}\frac{\beta k c I_{m}\left(\bar{k} r_{b}\right)}{2 \pi r_{b}^{m} \gamma}\left[K_{m-1}(\bar{k} \rho)+K_{m+1}(\bar{k} \rho)\right] \cos m\left(\theta-\theta_{b}\right) e^{-j k z} & \text { for } \rho>r_{b}, \\
-\frac{\beta k c K_{m}\left(\bar{k} r_{b}\right)}{2 \pi r_{b}^{m} \gamma}\left[I_{m-1}(\bar{k} \rho)+I_{m+1}(\bar{k} \rho)\right] \cos m\left(\theta-\theta_{b}\right) e^{-j k z} & \text { for } r_{b}>\rho,\end{cases}
\end{gathered}
$$

for $m>0$, where $k=2 \pi f / \beta c, \bar{k}=k / \gamma$, and $K_{m}(z)$ and $I_{m}(z)$ are the modified Bessel functions, respectively.

General solutions (especially $E_{z}, H_{\theta}$ ) inside the chamber for $m=0$ are expressed as 


$$
\begin{gathered}
E_{z}=E_{z}^{S}+\int_{-\infty}^{\infty} d h A(h) e^{-j h z} \frac{J_{0}(\Lambda \rho)}{J_{0}(\Lambda a)}, \\
H_{\theta}=H_{\theta}^{S}+\frac{j k \beta}{Z_{0}} \int_{-\infty}^{\infty} d h A(h) e^{-j h z} \frac{J_{1}(\Lambda \rho)}{\Lambda J_{0}(\Lambda a)},
\end{gathered}
$$

and those (especially $E_{z}, E_{\rho}, H_{\theta}, H_{z}$ ) for $m>0$ are

$$
\begin{aligned}
E_{z}= & i_{m}\left[E_{z}^{S}+\int_{-\infty}^{\infty} d h A(h) e^{-j h z} \frac{J_{m}(\Lambda \rho)}{J_{m}(\Lambda a)} \cos m\left(\theta-\theta_{b}\right)\right], \\
H_{\theta}= & i_{m}\left[H_{\theta}^{S}-\int_{-\infty}^{\infty} d h e^{-j h z} \frac{j h}{\Lambda^{2}}\left(\frac{m B(h) J_{m}(\Lambda \rho)}{\rho J_{m}(\Lambda a)}\right.\right. \\
& \left.\left.+\frac{k \beta}{Z_{0} h} A(h) \frac{\frac{\partial J_{m}(\Lambda \rho)}{\partial \rho}}{J_{m}(\Lambda a)}\right) \cos m\left(\theta-\theta_{b}\right)\right], \\
H_{z}= & \int_{-\infty}^{\infty} d h i_{m} B(h) e^{-j h z} \frac{J_{m}(\Lambda \rho)}{J_{m}(\Lambda a)} \sin m\left(\theta-\theta_{b}\right), \quad(\mathrm{A} 16) \\
E_{\theta}= & i_{m}\left[E_{\theta}^{S}+\int_{-\infty}^{\infty} d h e^{-j h z} \frac{j k \beta Z_{0}}{\Lambda^{2}}\left(B(h) \frac{\frac{\partial J_{m}(\Lambda \rho)}{\partial \rho}}{J_{m}(\Lambda a)}\right.\right. \\
+ & \left.\left.\frac{m h}{Z_{0} k \beta \rho} A(h) \frac{J_{m}(\Lambda \rho)}{J_{m}(\Lambda a)}\right) \sin m\left(\theta-\theta_{b}\right)\right],
\end{aligned}
$$

where $J_{m}(z)$ is the Bessel function and $\Lambda=\sqrt{k^{2} \beta^{2}-h^{2}}$.

\section{Impedance of a perfectly conductive chamber}

When a beam with the current density $j_{z}=\beta c[1-$ $\Theta(\rho-\sigma)] e^{-j k z} /\left(\pi \sigma^{2}\right)$, where $\Theta(x)$ is the step function, passes through the chamber, the source field (especially $\left.E_{z}\right)$ is expressed as

$$
\begin{aligned}
E_{z}^{S}= & \frac{j k c Z_{0} e^{-j k z}}{\pi \sigma^{2} \gamma^{2}}\left(\int_{0}^{\rho} d r_{b} r_{b} I_{0}\left(\bar{k} r_{b}\right) K_{0}(\bar{k} \rho)\right. \\
& \left.+\int_{\rho}^{\sigma} d r_{b} r_{b} K_{0}\left(\bar{k} r_{b}\right) I_{0}(\bar{k} \rho)\right) \\
= & \frac{j c Z_{0}}{\pi \gamma \sigma^{2}}\left(\frac{1}{\bar{k}}-\sigma I_{0}(\bar{k} \rho) K_{1}(\bar{k} \sigma)\right) e^{-j k z} \quad \text { for } \rho \leq \sigma \\
E_{z}^{S}= & \frac{j k c Z_{0} e^{-j k z}}{\pi \sigma^{2} \gamma^{2}} \int_{0}^{\sigma} d r_{b} r_{b} I_{0}\left(\bar{k} r_{b}\right) K_{0}(\bar{k} \rho) \\
= & \frac{j c Z_{0}}{\pi \sigma \gamma} I_{1}(\bar{k} \sigma) K_{0}(\bar{k} \rho) e^{-j k z} \quad \text { for } \rho \geq \sigma
\end{aligned}
$$

When the chamber is made of the perfectly conductive material, the longitudinal electric field inside the chamber is given by

$$
\begin{aligned}
E_{z}= & \frac{j c Z_{0}}{\pi \gamma \sigma^{2}}\left(\frac{1}{\bar{k}}-\sigma I_{0}(\bar{k} \rho) K_{1}(\bar{k} \sigma)\right) e^{-j k z} \\
& -\frac{j c Z_{0}}{\pi \sigma \gamma} I_{1}(\bar{k} \sigma) K_{0}(\bar{k} a) \frac{I_{0}(\bar{k} \rho)}{I_{0}(\bar{k} a)} e^{-j k z} \quad \text { for } \rho \geq \sigma,
\end{aligned}
$$

$$
\begin{aligned}
E_{z}= & \frac{j c Z_{0}}{\pi \sigma \gamma} I_{1}(\bar{k} \sigma) K_{0}(\bar{k} \rho) e^{-j k z} \\
& -\frac{j c Z_{0}}{\pi \sigma \gamma} I_{1}(\bar{k} \sigma) K_{0}(\bar{k} a) \frac{I_{0}(\bar{k} \rho)}{I_{0}(\bar{k} a)} e^{-j k z} \quad \text { for } \rho \geq \sigma .
\end{aligned}
$$

The coupling impedance $Z_{L}$ is defined as the average of the longitudinal electric field (normalized by the beam current) over the beam cross section. Then, we obtain

$$
\begin{aligned}
Z_{L}= & -\frac{j Z_{0}}{\beta \pi \sigma^{2} k}\left(1-\frac{2 K_{0}(\bar{k} a) I_{1}^{2}(\bar{k} \sigma)}{I_{0}(\bar{k} a)}\right. \\
& \left.-2 I_{1}(\bar{k} \sigma) K_{1}(\bar{k} \sigma)\right) \mathcal{L},
\end{aligned}
$$

where $\mathcal{L}$ is the length of the beam pipe.

When a beam with the azimuthal dependency of $j_{z}=$ $q \beta c \delta\left(\rho-r_{b}\right) \cos \theta e^{-j k z} / \pi r_{b}$ is running inside the chamber, the longitudinal electric field is given by

$$
\begin{aligned}
E_{z}= & i_{1} \frac{j k c Z_{0} I_{1}\left(\bar{k} r_{b}\right)}{\pi r_{b} \gamma^{2}}\left(K_{1}(\bar{k} \rho)-K_{1}(\bar{k} a) \frac{I_{1}(\bar{k} \rho)}{I_{1}(\bar{k} a)}\right) \\
& \times \cos \left(\theta-\theta_{b}\right) e^{-j k z} \text { for } \rho>r_{b},
\end{aligned}
$$

$$
\begin{aligned}
E_{z}= & i_{1} \frac{j k c Z_{0} I_{1}(\bar{k} \rho)}{\pi r_{b} \gamma^{2}}\left(K_{1}\left(\bar{k} r_{b}\right)-K_{1}(\bar{k} a) \frac{I_{1}\left(\bar{k} r_{b}\right)}{I_{1}(\bar{k} a)}\right) \\
& \times \cos \left(\theta-\theta_{b}\right) e^{-j k z} \quad \text { for } \rho<r_{b}
\end{aligned}
$$

where $i_{1}=q r_{b}$. Using the Panofsky-Wenzel theorem $[7,16]$, we can obtain the transverse wake forces from the longitudinal electric fields. The transverse impedance is thus given by

$$
Z_{T}=\frac{R k Z_{0}}{j \beta r_{b} \gamma^{3}}\left(K_{1}\left(\bar{k} r_{b}\right)-\frac{I_{1}\left(\bar{k} r_{b}\right)}{I_{1}(\bar{k} a)} K_{1}(\bar{k} a)\right)
$$

\section{Impedance of the resistive chamber with finite thickness}

\section{a. Longitudinal impedance}

General solutions (especially $E_{z}, H_{\theta}$ ) for $m=0$ are expressed as

$$
\begin{gathered}
E_{z}=E_{z}^{S}+A(k) e^{-j k z} I_{0}(\bar{k} \rho), \\
H_{\theta}=H_{\theta}^{S}+\frac{j \beta \gamma}{Z_{0}} A(k) e^{-j k z} I_{1}(\bar{k} \rho),
\end{gathered}
$$


inside the chamber $(\rho<a)$,

$$
\begin{aligned}
E_{z} & =e^{-j k z}\left[C_{1}(k) I_{0}\left(\nu_{2} \rho\right)+C_{2}(k) K_{0}\left(\nu_{2} \rho\right)\right], \\
H_{\theta} & =\frac{\sigma}{\nu_{2}} e^{-j k z}\left[C_{1}(k) I_{1}\left(\nu_{2} \rho\right)-C_{2}(k) K_{1}\left(\nu_{2} \rho\right)\right],
\end{aligned}
$$

inside the resistive material $(a<\rho<a+t)$, and

$$
\begin{gathered}
E_{z}=D_{1}(k) e^{-j k z} K_{0}(\bar{k} \rho), \\
H_{\theta}=-\frac{j \beta \gamma}{Z_{0}} D_{1}(k) e^{-j k z} K_{1}(\bar{k} \rho),
\end{gathered}
$$

for open space $(a+t<\rho)$, where $\nu_{2}=\sqrt{k^{2}+j k \beta Z_{0} \sigma_{c}}, t$ is the thickness of this chamber and $A(k), C_{1}(k), C_{2}(k)$, and $D_{1}(k)$ are arbitrary coefficients. From the matching conditions on each surface specified by $\rho=a$ and $\rho=a+t$, we solve the coefficient $A(k)$ as

$$
A(k)=\frac{j k c Z_{0} I_{0}\left(\bar{k} r_{b}\right) C_{3}}{2 \pi \gamma^{2}}
$$

where $C_{3}$ is given by Eq. (36).

Similarly to the perfectly conductive chamber case, when a beam with the current density $j_{z}=\beta c[1-\Theta(\rho-$ $\sigma)] e^{-j k z} /\left(\pi \sigma^{2}\right)$ passes through the chamber, $E_{z}$ inside the chamber is expressed as

$$
\begin{gathered}
E_{z}=\frac{j k c Z_{0} e^{-j k z}}{\pi \sigma^{2} \gamma^{2}}\left(\int_{0}^{\rho} d r_{b} r_{b} I_{0}\left(\bar{k} r_{b}\right) K_{0}(\bar{k} \rho)+\int_{\rho}^{\sigma} d r_{b} r_{b} K_{0}\left(\bar{k} r_{b}\right) I_{0}(\bar{k} \rho)+C_{3} \int_{0}^{\sigma} d r_{b} r_{b} I_{0}\left(\bar{k} r_{b}\right) I_{0}(\bar{k} \rho)\right) \\
=\frac{j c Z_{0}}{\pi \gamma \sigma^{2}}\left(\frac{1}{\bar{k}}-\sigma I_{0}(\bar{k} \rho) K_{1}(\bar{k} \sigma)+C_{3} \sigma I_{1}(\bar{k} \sigma) I_{0}(\bar{k} \rho)\right) e^{-j k z} \quad \text { for } \rho \leq \sigma, \\
E_{z}=\frac{j k c Z_{0} e^{-j k z}}{\pi \sigma^{2} \gamma^{2}}\left(\int_{0}^{\sigma} d r_{b} r_{b} I_{0}\left(\bar{k} r_{b}\right) K_{0}(\bar{k} \rho)+C_{3} \int_{0}^{\sigma} d r_{b} r_{b} I_{0}\left(\bar{k} r_{b}\right) I_{0}(\bar{k} \rho)\right) \\
=\frac{j c Z_{0} I_{1}(\bar{k} \sigma)}{\pi \sigma \gamma}\left[K_{0}(\bar{k} \rho)+C_{3} I_{0}(\bar{k} \rho)\right] e^{-j k z} \quad \text { for } \rho \geq \sigma .
\end{gathered}
$$

Since the coupling impedance $Z_{L}$ is defined as the average of the longitudinal electric field (normalized by the beam current) over the beam cross section, we obtain the final expression of the resistive-wall impedance by extracting the space charge impedance Eq. (A22):

$$
Z_{L}=-\frac{2 j Z_{0} I_{1}^{2}(\bar{k} \sigma)}{\beta \pi \sigma^{2} k}\left(\frac{K_{0}(\bar{k} a)}{I_{0}(\bar{k} a)}+C_{3}\right) \mathcal{L} .
$$

By taking a limit of $\sigma \rightarrow 0$ and replacing $\mathcal{L}$ by the longitudinal length of the chamber $g$, we reproduce Eq. (35).

\section{b. Transverse impedance}

General solutions (especially $E_{z}, E_{\theta}, H_{z}, H_{\theta}$ ) for $m=1$ are expressed as

$$
\begin{gathered}
E_{z}=i_{1}\left[E_{z}^{S}+A(k) I_{1}(\bar{k} \rho) \cos \left(\theta-\theta_{b}\right) e^{-j k z}\right], \\
H_{\theta}=i_{1}\left[H_{\theta}^{S}+\frac{j \gamma}{\bar{k}}\left(\frac{B(k) I_{1}(\bar{k} \rho)}{\rho}+\frac{\beta \bar{k} A(k)}{Z_{0}} I_{1}^{\prime}(\bar{k} \rho)\right)\right. \\
\left.\quad \times \cos \left(\theta-\theta_{b}\right) e^{-j k z}\right] \\
H_{z}=i_{1} B(k) I_{1}(\bar{k} \rho) \sin \left(\theta-\theta_{b}\right) e^{-j k z},
\end{gathered}
$$

$$
\begin{aligned}
E_{\theta}= & i_{1}\left[E_{\theta}^{S}-\frac{j \beta \gamma Z_{0}}{\bar{k}}\left(\bar{k} B(k) I_{1}^{\prime}(\bar{k} \rho)+\frac{A(k)}{Z_{0} \beta \rho} I_{1}(\bar{k} \rho)\right)\right. \\
& \left.\times \sin \left(\theta-\theta_{b}\right) e^{-j k z}\right],
\end{aligned}
$$

inside the chamber $(\rho<a)$,

$$
E_{z}=\left[C_{3}(k) I_{1}\left(\nu_{2} \rho\right)+C_{4}(k) K_{1}\left(\nu_{2} \rho\right)\right] \cos \left(\theta-\theta_{b}\right) e^{-j k z},
$$

$$
\begin{aligned}
H_{\theta}= & \frac{j k}{\nu_{2}^{2}}\left(\frac{C_{1}(k) I_{1}\left(\nu_{2} \rho\right)+C_{2}(k) K_{1}\left(\nu_{2} \rho\right)}{\rho}\right. \\
& \left.+\frac{\sigma \nu_{2}\left[C_{3}(k) I_{1}^{\prime}\left(\nu_{2} \rho\right)+C_{4}(k) K_{1}^{\prime}\left(\nu_{2} \rho\right)\right]}{j k}\right) \\
& \times \cos \left(\theta-\theta_{b}\right) e^{-j k z}, \\
H_{z}= & {\left[C_{1}(k) I_{1}\left(\nu_{2} \rho\right)+C_{2}(k) K_{1}\left(\nu_{2} \rho\right)\right] \sin \left(\theta-\theta_{b}\right) e^{-j k z}, }
\end{aligned}
$$

$$
\begin{aligned}
E_{\theta}= & -\frac{j k}{\nu_{2}^{2}}\left(\frac{C_{3}(k) I_{1}\left(\nu_{2} \rho\right)+C_{4}(k) K_{1}\left(\nu_{2} \rho\right)}{\rho}\right. \\
& \left.+\beta Z_{0} \nu_{2}\left[C_{1}(k) I_{1}^{\prime}\left(\nu_{2} \rho\right)+C_{2}(k) K_{1}^{\prime}\left(\nu_{2} \rho\right)\right]\right) \\
& \times \cos \left(\theta-\theta_{b}\right) e^{-j k z},
\end{aligned}
$$

inside the resistive material $(a<\rho<a+t)$, and 


$$
\begin{aligned}
& E_{z}=i_{1} D_{4}(k) K_{1}(\bar{k} \rho) \cos \left(\theta-\theta_{b}\right) e^{-j k z} \\
H_{\theta}= & \frac{j \gamma}{\bar{k}}\left(\frac{i_{1} D_{2}(k) K_{1}(\bar{k} \rho)}{\rho}+\frac{\beta \bar{k} i_{1} D_{4}(k)}{Z_{0}} K_{1}^{\prime}(\bar{k} \rho)\right) \\
& \times \cos \left(\theta-\theta_{b}\right) e^{-j k z} \\
& H_{z}=i_{1} D_{2}(k) K_{1}(\bar{k} \rho) \sin \left(\theta-\theta_{b}\right) e^{-j k z} \\
E_{\theta}= & -\frac{j \beta \gamma Z_{0}}{\bar{k}}\left(\bar{k} i_{1} D_{2}(k) K_{1}^{\prime}(\bar{k} \rho)+\frac{i_{1} D_{4}(k)}{Z_{0} \beta \rho} K_{1}(\bar{k} \rho)\right) \\
& \times \sin \left(\theta-\theta_{b}\right) e^{-j k z},
\end{aligned}
$$

for open space $(a+t<\rho), A(k), B(k), C_{1}(k), C_{2}(k)$, $C_{3}(k), C_{4}(k), D_{2}(k)$, and $D_{4}(k)$ are arbitrary coefficients, which are determined by boundary conditions.
Using the Panofsky-Wenzel theorem and extracting the transverse impedance of perfectly conducting chamber [Eq. (A25)], we obtain the expression of the transverse impedance of the resistive-wall chamber as

$$
Z_{T}=-\frac{g A(k)}{2 \gamma c \beta}+\frac{g k Z_{0} I_{1}\left(\bar{k} r_{b}\right) K_{1}(\bar{k} a)}{j 2 \pi \beta r_{b} \gamma^{3} I_{1}(\bar{k} a)} .
$$

After taking a limit at $r_{b} \rightarrow 0$, we reproduce Eq. (13) in Ref. [11]:

$$
Z_{T}=\frac{j g Z_{0} \bar{k}^{2} K_{1}(\bar{k} a) E_{2}\left(\alpha_{2}-1\right)}{4 \pi \beta \gamma^{2} I_{1}(\bar{k} a)},
$$

where the parameters $E_{2}$ and $\alpha_{2}$ are two parameters out of four, $\alpha_{2}, \eta_{2}, E_{2}$, and $G_{2}$, which have to be found by solving the following linear equations:

$$
\begin{gathered}
\frac{\left(\nu_{2}^{2}-\bar{k}^{2}\right)}{\bar{k} \beta a \nu_{2}} E_{2}\left(1-\alpha_{2}\right)+\left(\nu_{2} \frac{I_{1}^{\prime}(\bar{k} a)}{I_{1}(\bar{k} a)}-\bar{k} \frac{K_{1}^{\prime}\left(\nu_{2} a\right)}{K_{1}\left(\nu_{2} a\right)}\right) G_{2}-\left(\nu_{2} \frac{I_{1}^{\prime}(\bar{k} a)}{I_{1}(\bar{k} a)}-\bar{k} \frac{I_{1}^{\prime}\left(\nu_{2} a\right)}{I_{1}\left(\nu_{2} a\right)}\right) G_{2} \eta_{2}=0, \\
\left(\beta \nu_{2} \frac{I_{1}^{\prime}(\bar{k} a)}{I_{1}(\bar{k} a)}+j \frac{Z_{0} \sigma_{c}}{\gamma} \frac{K_{1}^{\prime}\left(\nu_{2} a\right)}{K_{1}\left(\nu_{2} a\right)}\right) E_{2}-\left(\beta \nu_{2} \frac{I_{1}^{\prime}(\bar{k} a)}{I_{1}(\bar{k} a)}+j \frac{Z_{0} \sigma_{c}}{\gamma} \frac{I_{1}^{\prime}\left(\nu_{2} a\right)}{I_{1}\left(\nu_{2} a\right)}\right) E_{2} \alpha_{2}+\frac{\left(\nu_{2}^{2}-\bar{k}^{2}\right)}{\nu_{2} \bar{k} a} G_{2}\left(1-\eta_{2}\right) \\
=-\beta \nu_{2}\left(\frac{K_{1}^{\prime}(\bar{k} a)}{K_{1}(\bar{k} a)}-\frac{I_{1}^{\prime}(\bar{k} a)}{I_{1}(\bar{k} a)}\right), \\
\left(\bar{k}^{2}-\nu_{2}^{2}\right) \\
\overline{\bar{k} \nu_{2} \beta(a+t)} \frac{K_{1}\left[\nu_{2}(a+t)\right]}{K_{1}\left(\nu_{2} a\right)} E_{2}-\frac{\left(\bar{k}^{2}-\nu_{2}^{2}\right)}{\bar{k} \nu_{2} \beta(a+t)} \frac{I_{1}\left[\nu_{2}(a+t)\right]}{I_{1}\left(\nu_{2} a\right)} E_{2} \alpha_{2}+\left(\bar{k} \frac{K_{1}^{\prime}\left[\nu_{2}(a+t)\right]}{K_{1}\left(\nu_{2} a\right)}-\nu_{2} \frac{K_{1}\left[\nu_{2}(a+t)\right]}{K_{1}\left(\nu_{2} a\right)} \frac{K_{1}^{\prime}[\bar{k}(a+t)]}{K_{1}[\bar{k}(a+t)]}\right) G_{2}\left(\bar{k}_{1}^{\prime}\left[\nu_{2}(a+t)\right]\right. \\
-\left(\nu_{2} \frac{I_{1}\left[\nu_{2}(a+t)\right]}{I_{1}\left(\nu_{2} a\right)} \frac{K_{1}^{\prime}[\bar{k}(a+t)]}{K_{1}[\bar{k}(a+t)]} G_{2} \eta_{2}=0, \quad\right. \text { (A52) } \\
\left.+\beta \frac{K_{1}^{\prime}[\bar{k}(a+t)]}{K_{1}[\bar{k}(a+t)]} \frac{K_{1}\left[\nu_{2}(a+t)\right]}{K_{1}\left(\nu_{2} a\right)}+j \frac{Z_{0} \sigma_{c}}{\gamma} \frac{K_{1}^{\prime}\left[\nu_{2}(a+t)\right]}{K_{1}\left(\nu_{2} a\right)}\right) E_{2} \\
+\left(\nu_{2} \beta \frac{K_{1}^{\prime}[\bar{k}(a+t)]}{K_{1}[\bar{k}(a+t)]} \frac{I_{1}\left[\nu_{2}(a+t)\right]}{I_{1}\left(\nu_{2} a\right)}+j \frac{Z_{0} \sigma_{c}}{\gamma} \frac{I_{1}^{\prime}\left[\nu_{2}(a+t)\right]}{I_{1}\left(\nu_{2} a\right)}\right) E_{2} \alpha+\frac{\left(\bar{k}^{2}-\nu_{2}^{2}\right)}{\nu_{2} \bar{k}(a+t)} \frac{K_{1}\left[\nu_{2}(a+t)\right]}{K_{1}\left(\nu_{2} a\right)} G_{2} \\
-\frac{\left(\bar{k}^{2}-\nu_{2}^{2}\right)}{\nu_{2} \bar{k}(a+t)} \frac{I_{1}\left[\nu_{2}(a+t)\right]}{I_{1}\left(\nu_{2} a\right)} G_{2} \eta_{2}=0, \quad(\mathrm{~A} 53)
\end{gathered}
$$

$\nu_{2}=\sqrt{k^{2}+j k \beta Z_{0} \sigma_{c}}$, the prime of $I_{n}^{\prime}(z)$ and $K_{n}^{\prime}(z)$ means the differential by $z$.

\section{APPENDIX B: THE EXTERNAL FIELD PRODUCED BY A SLOT}

We construct the external field by superposition of basic sets of cylindrical waves. We only consider outward traveling waves (the Hankel function of the second kind). For any field component $E_{\alpha}(\rho, \theta, z)$, we can write

$$
E_{\alpha}(\rho, \theta, z)=\int_{-\infty}^{\infty} \mathcal{E}_{\alpha}(\rho, \theta, z) d h .
$$

The Fourier series of electromagnetic fields are formally written as follows:

$$
\begin{aligned}
\mathcal{E}_{\rho}= & \sum_{n=-\infty}^{\infty}\left[-j h a_{n} \frac{\partial H_{n}^{(2)}(\Lambda \rho)}{\partial \rho}\right. \\
& \left.-\frac{n k \beta Z_{0}}{\rho} b_{n} H_{n}^{(2)}(\Lambda \rho)\right] e^{-j n \theta} e^{-j h z}, \\
\mathcal{E}_{\theta}= & \sum_{n=-\infty}^{\infty}\left[-\frac{n h}{\rho} a_{n} H_{n}^{(2)}(\Lambda \rho)\right. \\
& \left.+j k \beta Z_{0} b_{n} \frac{\partial H_{n}^{(2)}(\Lambda \rho)}{\partial \rho}\right] e^{-j n \theta} e^{-j h z},
\end{aligned}
$$




$$
\begin{aligned}
\mathcal{E}_{z}= & \sum_{n=-\infty}^{\infty} \Lambda^{2} a_{n} H_{n}^{(2)}(\Lambda \rho) e^{-j n \theta} e^{-j h z}, \\
\mathcal{H}_{\rho}= & \sum_{n=-\infty}^{\infty}\left[\frac{n k \beta}{Z_{0} \rho} a_{n} H_{n}^{(2)}(\Lambda \rho)\right. \\
& \left.-j h b_{n} \frac{\partial H_{n}^{(2)}(\Lambda \rho)}{\partial \rho}\right] e^{-j n \theta} e^{-j h z}, \\
\mathcal{H}_{\theta}= & \sum_{n=-\infty}^{\infty}\left[-j \frac{k \beta}{Z_{0}} a_{n} \frac{\partial H_{n}^{(2)}(\Lambda \rho)}{\partial \rho}\right. \\
& \left.-\frac{n h}{\rho} b_{n} H_{n}^{(2)}(\Lambda \rho)\right] e^{-j n \theta} e^{-j h z}
\end{aligned}
$$

$$
\mathcal{H}_{z}=\sum_{n=-\infty}^{\infty} \Lambda^{2} b_{n} H_{n}^{(2)}(\Lambda \rho) e^{-j n \theta} e^{-j h z},
$$

where $H_{n}^{(2)}(z)$ is the Hankel function of the second kind.

The tangential electric field in the slot will have both $\theta$ and $z$ components in general. We express them using the prescribed functions $f_{1}(\theta, z)$ and $f_{2}(\theta, z)$ as

$$
\begin{aligned}
& f_{1}(\theta, z)= \begin{cases}E_{\theta}(a, \theta, z) & \text { on the slot }\left(\theta_{1} \leq \theta \leq \theta_{2} ; z_{1} \leq z \leq z_{2}\right) \\
0 & \text { outside the slot, }\end{cases} \\
& f_{2}(\theta, z)= \begin{cases}E_{z}(a, \theta, z) & \text { on the slot }\left(\theta_{1} \leq \theta \leq \theta_{2} ; z_{1} \leq z \leq z_{2}\right) \\
0 & \text { outside the slot. }\end{cases}
\end{aligned}
$$

The Fourier expansion coefficients are expressed as

$$
\begin{aligned}
a_{n}= & \frac{1}{4 \pi^{2} \Lambda^{2} H_{n}^{(2)}(\Lambda a)} \int_{z_{1}}^{z_{2}} d \xi \int_{\theta_{1}(\xi)}^{\theta_{2}(\xi)} d \beta f_{2}(\beta, \xi) e^{j n \beta} e^{j h \xi}, \\
b_{n}= & -\frac{j n h}{4 \pi^{2} k \beta Z_{0} a \Lambda^{2}\left[\partial H_{n}^{(2)}(\Lambda a) / \partial a\right]} \\
& \times \int_{z_{1}}^{z_{2}} d \xi \int_{\theta_{1}(\xi)}^{\theta_{2}(\xi)} d \beta f_{2}(\beta, \xi) e^{j n \beta} e^{j h \xi} \\
& -\frac{j}{4 \pi^{2} k \beta Z_{0}\left[\partial H_{n}^{(2)}(\Lambda a) / \partial a\right]} \\
& \times \int_{z_{1}}^{z_{2}} d \xi \int_{\theta_{1}(\xi)}^{\theta_{2}(\xi)} d \beta f_{1}(\beta, \xi) e^{j n \beta} e^{j h \xi} .
\end{aligned}
$$

We consider the circumferential slot, which goes all the way around the cylindrical chamber with the width of $2 w$ $\left[z_{1}=-w, z_{2}=w, \theta_{1}(z)=0, \theta_{2}(z)=2 \pi\right]$ (which is much smaller than the chamber radius). Suppose that the tangential electric field in the slot has only the $z$ component, which is independent of $\theta$. Namely,

$$
f_{1}(\theta, z)=0 ; \quad f_{2}(\theta, z)=\frac{\tilde{V}}{2 w} .
$$

Then, the nonzero coefficient is only for $n=0$ and is readily given by

$$
a_{0}=\frac{\tilde{V} \sin h w / h w}{2 \pi \Lambda^{2} H_{0}^{(2)}(\Lambda a)} .
$$

The general expression of the fields can be written as

$$
\begin{gathered}
E_{z}=\frac{\tilde{V}}{2 \pi} \int_{-\infty}^{\infty} \frac{H_{0}^{(2)}(\Lambda \rho)}{H_{0}^{(2)}(\Lambda a)} \frac{\sin h w}{h w} e^{-j h z} d h, \\
H_{\theta}=j \frac{\beta k}{Z_{0}} \frac{\tilde{V}}{2 \pi} \int_{-\infty}^{\infty} \frac{H_{1}^{(2)}(\Lambda \rho)}{\Lambda H_{0}^{(2)}(\Lambda a)} \frac{\sin h w}{h w} e^{-j h z} d h .
\end{gathered}
$$

If the tangential electric field in the slot has both $z$ and $\theta$ components (especially the $n=1$ case), we can get

$$
f_{1}(\theta, z)=\frac{i_{1} V_{1} \pi}{w} \sin \theta ; \quad f_{2}(\theta, z)=\frac{i_{1} V_{2} \pi}{w} \cos \theta .
$$

Consequently, the only nonzero coefficients are

$$
\begin{gathered}
a_{ \pm 1}=\frac{i_{1} V_{2} \sin h w / h w}{2 \Lambda^{2} H_{ \pm 1}^{(2)}(\Lambda a)}, \\
b_{ \pm 1}= \pm\left(i_{1} V_{1}-j \frac{h i_{1} V_{2}}{a \Lambda^{2}}\right) \frac{\sin h w / h w}{2 k \beta Z_{0}\left[\partial H_{ \pm 1}^{(2)}(\Lambda a) / \partial a\right]} .
\end{gathered}
$$


Finally, we reach the general expression of the fields as

$$
\begin{aligned}
E_{z}= & i_{1} V_{2} \int_{-\infty}^{\infty} d h \frac{H_{1}^{(2)}(\Lambda \rho)}{H_{1}^{(2)}(\Lambda a)} \frac{\sin h w}{h w} e^{-j h z} \cos \theta, \quad \text { (B19) } \\
H_{\theta}= & i_{1} V_{2} \int_{-\infty}^{\infty} d h\left(-j \frac{k \beta}{Z_{0}} \frac{\frac{\partial H_{1}^{(2)}(\Lambda \rho)}{\Lambda^{2} H_{1}^{(2)}(\Lambda a)}}{}\right. \\
& \left.+j \frac{h^{2}}{\rho} \frac{H_{1}^{(2)}(\Lambda \rho)}{Z_{0} k \beta a \Lambda^{2} \frac{\partial H_{1}^{(2)}(\Lambda a)}{\partial a}}\right) \frac{\sin h w}{h w} e^{-j h z} \cos \theta-i_{1} V_{1} \\
& \times \int_{-\infty}^{\infty} d h \frac{h}{\rho} \frac{H_{1}^{(2)}(\Lambda \rho)}{Z_{0} k \beta \frac{\partial H_{1}^{(2)}(\Lambda a)}{\partial a}} \frac{\sin h w}{h w} e^{-j h z} \cos \theta, \quad(\mathrm{B} 20) \\
H_{z}= & -\int_{-\infty}^{\infty} d h j \Lambda^{2}\left(i_{1} V_{1}-j \frac{i_{1} V_{2} h}{a \Lambda^{2}}\right) \frac{H_{1}^{(2)}(\Lambda \rho)}{Z_{0} k \beta \frac{\partial H_{1}^{(2)}(\Lambda a)}{\partial a}} \\
& \times \frac{\sin h w}{h w} e^{-j h z} \sin \theta,
\end{aligned}
$$

$$
\begin{aligned}
E_{\theta}= & i_{1} V_{2} \int_{-\infty}^{\infty} d h\left(j \frac{h}{\rho \Lambda^{2}} \frac{H_{1}^{(2)}(\Lambda \rho)}{H_{1}^{(2)}(\Lambda a)}-j \frac{h}{a \Lambda^{2}} \frac{\frac{\partial H_{1}^{(2)}(\Lambda \rho)}{\partial H_{1}^{(2)}(\Lambda a)}}{\partial a}\right) \\
& \times \frac{\sin h w}{h w} e^{-j h z} \sin \theta \\
& +i_{1} V_{1} \int_{-\infty}^{\infty} d h \frac{\frac{\partial H_{1}^{(2)}(\Lambda \rho)}{\partial \rho}}{\frac{\partial H_{1}^{(2)}(\Lambda a)}{\partial a}} \frac{\sin h w}{h w} e^{-j h z} \sin \theta .
\end{aligned}
$$

\section{APPENDIX C: EXPANSION FORMULAS OF THE BESSEL AND HANKEL FUNCTIONS}

The Bessel functions have the following relationships:

$$
\begin{gathered}
\frac{J_{n}^{\prime}(z)}{J_{n}(z)}=\frac{n}{z}+\sum_{k=1}^{\infty} \frac{2 z}{z^{2}-j_{n, k}^{2}}, \\
\frac{J_{1}(z)}{z J_{1}^{\prime}(z)}=\sum_{k=1}^{\infty} \frac{2}{z^{2}-j_{1, k}^{\prime 2}} \frac{J_{1}\left(j_{1, k}^{\prime}\right)}{J_{1}^{\prime \prime}\left(j_{1, k}^{\prime}\right)},
\end{gathered}
$$

where $j_{n, k}$ are the $k$ th zeros of $J_{n}(z)$ and $j_{1, k}^{\prime}$ are the $k$ th zeros of $J_{1}^{\prime}(z)$ [17].

Similar formulas can be obtained for the Hankel functions after some manipulations as follows:

$$
\begin{gathered}
\frac{1}{\sqrt{z}} \frac{H_{1}^{(2)}(\sqrt{z})}{H_{0}^{(2)}(\sqrt{z})}=\int_{0}^{\infty} d \zeta \frac{2}{\pi^{2} \zeta(\zeta+z) H_{0}^{(1)}\left(e^{(\pi / 2) j} \sqrt{\zeta}\right) H_{0}^{(2)}\left(e^{(\pi / 2) j} \sqrt{\zeta}\right)} \\
\frac{1}{\sqrt{z}} \frac{H_{1}^{\prime(2)}(\sqrt{z})}{H_{1}^{(2)}(\sqrt{z})}=-\frac{1}{z}-\int_{0}^{\infty} d \zeta \frac{2}{\pi^{2} \zeta(\zeta+z) H_{1}^{(1)}\left(e^{(\pi / 2) j} \sqrt{\zeta}\right) H_{1}^{(2)}\left(e^{(\pi / 2) j} \sqrt{\zeta}\right)}, \\
\frac{1}{\sqrt{z}} \frac{H_{1}^{(2)}(\sqrt{z})}{H_{1}^{\prime(2)}(\sqrt{z})}=\frac{2 H_{1}^{(2)}\left(h_{1,0}^{\prime}\right)}{\left(z-h_{1,0}^{\prime 2}\right) H_{1}^{\prime \prime(2)}\left(h_{1,0}^{\prime}\right)}+\int_{0}^{\infty} d \zeta \frac{2}{\pi^{2} \zeta(\zeta+z) H_{1}^{\prime(1)}\left(e^{(\pi / 2) j} \sqrt{\zeta}\right) H_{1}^{\prime(2)}\left(e^{(\pi / 2) j} \sqrt{\zeta}\right)},
\end{gathered}
$$

where $h_{1,0}^{\prime}=0.501184+j 0.643545$ : the 0th zero of $H_{1}^{\prime(2)}(z)$ (the differential of the Hankel function of the second kind), and $H_{\nu}^{(1)}(z)$ is the Hankel function of the first kind.

Let us prove the above formulas in the following way. Let us consider the following form of the integral:

$$
\frac{1}{2 \pi j} \oint \frac{F(\zeta)}{\sqrt{\zeta}(\zeta-z)} d \zeta
$$

First we take $F(\zeta)$ as

$$
F(\zeta)=\frac{H_{1}^{(2)}(\sqrt{\zeta})}{H_{0}^{(2)}(\sqrt{\zeta})},
$$

to prove Eq. (C3). We choose the path of the integral as shown in Fig. 10. The contribution of the integral from the infinity vanishes since $F(\zeta)$ approaches 1 as $|\zeta| \rightarrow \infty$. The contribution from $C_{\epsilon}$ approaches zero as $\epsilon \rightarrow 0$. The contribution from the poles except $\zeta=z$ vanishes because the Hankel function $H_{0}^{(2)}(z)$ has no poles in $-\pi / 2<z<\pi / 2$, which corresponds to $-\pi<\zeta<\pi$. Then, the following relationship can be found:

$$
\begin{aligned}
\frac{H_{1}^{(2)}(\sqrt{z})}{\sqrt{z} H_{0}^{(2)}(\sqrt{z})}= & \frac{1}{2 \pi} \int_{0}^{\infty} \frac{d \zeta}{\sqrt{\zeta}(\zeta+z)}\left[\frac{H_{1}^{(2)}\left(e^{(\pi / 2) j} \sqrt{\zeta}\right)}{H_{0}^{(2)}\left(e^{(\pi / 2) j} \sqrt{\zeta}\right)}\right. \\
& \left.+\frac{H_{1}^{(2)}\left(e^{-(\pi / 2) j} \sqrt{\zeta}\right)}{H_{0}^{(2)}\left(e^{-(\pi / 2) j} \sqrt{\zeta}\right)}\right] .
\end{aligned}
$$

We now derive Eq. (C3) by using the relationships

$$
H_{0}^{(2)}\left(e^{-\pi j} z\right)=-H_{0}^{(1)}(z)
$$




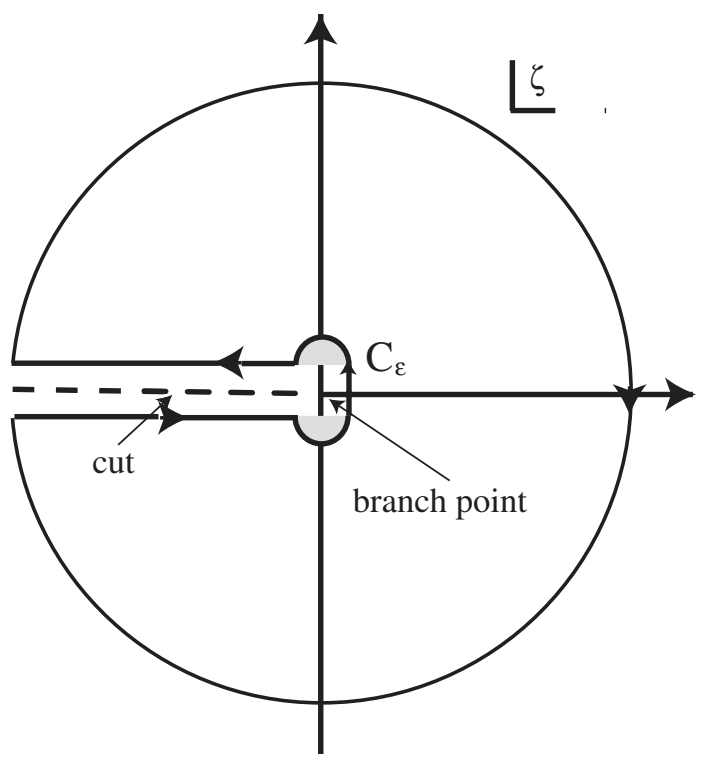

FIG. 10. The path of integration in Eq. (C6) in order to prove Eqs. (C3)-(C5). The horizontal and the vertical axes are the real and the imaginary axes, respectively.

$$
\begin{gathered}
H_{1}^{(2)}\left(e^{-\pi j} z\right)=H_{1}^{(1)}(z), \\
H_{0}^{(1)}(z) H_{1}^{(2)}(z)-H_{1}^{(1)}(z) H_{0}^{(2)}(z)=\frac{4 j}{\pi z},
\end{gathered}
$$

which can be found in Ref. [18].

Similarly, in order to prove Eq. (C4), we take $F(\zeta)$ as

$$
F(\zeta)=\frac{H_{1}^{\prime(2)}(\sqrt{\zeta})}{H_{1}^{(2)}(\sqrt{\zeta})}
$$

In this case, the contribution from the circle $C_{\epsilon}$ does not vanish. The following relationships can be found:

$$
\begin{aligned}
\frac{H_{1}^{\prime(2)}(\sqrt{z})}{\sqrt{z} H_{1}^{(2)}(\sqrt{z})}= & -\frac{1}{z}+\frac{1}{2 \pi} \int_{0}^{\infty} \frac{d \zeta}{\sqrt{\zeta}(\zeta+z)} \\
& \times\left[\frac{H_{1}^{\prime(2)}\left(e^{(\pi / 2) j} \sqrt{\zeta}\right)}{H_{1}^{(2)}\left(e^{(\pi / 2) j} \sqrt{\zeta}\right)}+\frac{H_{1}^{\prime(2)}\left(e^{-(\pi / 2) j} \sqrt{\zeta}\right)}{H_{1}^{(2)}\left(e^{-(\pi / 2) j} \sqrt{\zeta}\right)}\right]
\end{aligned}
$$

We can derive Eq. (C4) using Eq. (C10) and the relationships

$$
\begin{gathered}
H_{1}^{\prime(2)}\left(e^{-\pi j} z\right)=-H_{1}^{\prime(1)}(z), \\
H_{1}^{(1)}(z) H_{1}^{\prime(2)}(z)-H_{1}^{\prime(1)}(z) H_{1}^{(2)}(z)=-\frac{4 j}{\pi z} .
\end{gathered}
$$

For proof of Eq. (C5), we take $F(\zeta)$ as

$$
F(\zeta)=\frac{H_{1}^{(2)}(\sqrt{\zeta})}{H_{1}^{\prime(2)}(\sqrt{\zeta})}
$$

The contribution from the poles of the Hankel functions does not vanish in this case, while that from the circle $C_{\epsilon}$ vanishes. Then, we can obtain the following relationships:

$$
\begin{aligned}
\frac{1}{\sqrt{z}} \frac{H_{1}^{(2)}(\sqrt{z})}{H_{1}^{(2)}(\sqrt{z})}= & \frac{2 H_{1}^{(2)}\left(h_{1,0}^{\prime}\right)}{\left(z-h_{1,0}^{\prime 2}\right) H_{1}^{\prime(2)}\left(h_{1,0}^{\prime}\right)}+\frac{1}{2 \pi} \int_{0}^{\infty} \frac{d \zeta}{\sqrt{\zeta}(\zeta+z)} \\
& \times\left[\frac{H_{1}^{(2)}\left(e^{(\pi / 2) j} \sqrt{\zeta}\right)}{H_{1}^{\prime(2)}\left(e^{(\pi / 2) j} \sqrt{\zeta}\right)}+\frac{H_{1}^{(2)}\left(e^{-(\pi / 2) j} \sqrt{\zeta}\right)}{H_{1}^{(2)}\left(e^{-(\pi / 2) j} \sqrt{\zeta}\right)}\right]
\end{aligned}
$$

Equation (C5) can be proved by using Eqs. (C10), (C14), and (C15).

[1] M. Ivanyan and V. Tsakanov, in Proceedings of the 8th European Particle Accelerator Conference, Paris, 2002 (EPS-IGA and CERN, Geneva, 2002), p. 1511.

[2] S. Krinsky, B. Podobedov, and R. L. Gluckstern, Phys. Rev. ST Accel. Beams 7, 114401 (2004).

[3] G. Stupakov, Phys. Rev. ST Accel. Beams 8, 044401 (2005).

[4] R. L. Gluckstern and B. Zotter, Report No. CERN-AB2008-045, 2008.

[5] J. Laslett, K. Neil, and A. Sessler, Rev. Sci. Instrum. 36, 436 (1965).

[6] B. W. Zotter and S. A. Kheifets, Impedances and Wakes in High-Energy Particle Accelerators (World Scientific, Singapore, 1998).

[7] A. W. Chao, Physics of Collective Beam Instabilities in High Energy Accelerators (Wiley, New York, 1993).

[8] Handbook of Accelerator Physics and Engineering, edited by A.W. Chao and M. Tigner (World Scientific, Singapore, 1999).

[9] A. Burov and V. Lebedev, in Proceedings of the 8th European Particle Accelerator Conference, Paris, 2002 (Ref. [1]), p. 1452.

[10] E. Metral, B. Zotter, and B. Salvant, in Proceedings of the 2007 Particle Accelerator Conference, Albuquerque, New Mexico, 2007 (IEEE, Albuquerque, New Mexico, 2007), p. 4216.

[11] E. Metral, Report No. CERN-AB-2005-084, 2005.

[12] Y. Shobuda, Y. H. Chin, and K. Takata, Phys. Rev. ST Accel. Beams 10, 044403 (2007).

[13] S. Silver and W. K. Saunders, J. Appl. Phys. 21, 153 (1950).

[14] See, e.g., J. K. Jackson, Classical Electrodynamics (Wiley, New York, 1998), p. 312.

[15] K. Bane and M. Sands, Part. Accel. 25, 73 (1990).

[16] W. Panofsky and W. Wenzel, Rev. Sci. Instrum. 27, 967 (1956).

[17] R. L. Gluckstern and R. Li, Part. Accel. 29, 159 (1990).

[18] M. Abramowitz and I. Stegun, Handbook of Mathematical Functions-With Formulas, Graphs, and Mathematical Tables (Dover, New York, 1974). 